\title{
CIRCLE ACTIONS ON SIMPLY CONNECTED 4-MANIFOLDS
} BY

\author{
RONALD FINTUSHEL
}

\begin{abstract}
Locally smooth $S^{1}$-actions on simply connected 4-manifolds are studied in terms of their weighted orbit spaces. An equivariant classification theorem is proved, and the weighted orbit space is used to compute the quadratic form of a given simply connected 4 -manifold with $S^{1}$-action. This is used to show that a simply connected 4-manifold which admits a locally smooth $S^{1}$-action must be homotopy equivalent to a connected sum of copies of $S^{4}, C P^{2},-C P^{2}$, and $S^{2} \times S^{2}$.
\end{abstract}

1. Introduction. In this article we investigate locally smooth actions of the circle group $S^{1}$ on simply connected 4-manifolds. Our techniques are essentially geometric and are motivated by the work of P. Orlik and F. Raymond [9], [10], and H. Seifert [11]. To each locally smooth $S^{1}$-action on an oriented simply connected closed 4-manifold we associate the orbit space "weighted" with certain isotropy information. $\$ \S 3-6$ are devoted to the equivariant classification of these actions. Two such actions are orientation-preserving equivariantly homeomorphic if and only if their weighted orbit spaces are isomorphic. Furthermore, each legally weighted simply connected 3-manifold gives rise to an $S^{1}$-action on a simply connected 4-manifold which is constructed by means of equivariant plumbing and other pasting techniques. This gives a complete account of such actions and generalizes results of [4]. In the case of an action which is free except for a finite fixed point set, these results follow from work of Church and Lamotke [3].

In $\$ 7$ we turn to the question of extending $S^{1}$-actions on simply connected 4-manifolds to $T^{2}$-actions, and it is shown that an $S^{1}$-action extends if and only if the weighted orbit space has a relatively simple format.

In $\$ 8$ the information contained in the weighted orbit space is used to give a recipe for the computation of the quadratic form of a simply connected 4-manifold with given $S^{1}$-action. The main result of this section is that a simply connected 4-manifold which admits a locally smooth $S^{1}$-action is homotopy equivalent to a connected sum of copies of $S^{4}, C P^{2},-C P^{2}$, and $S^{2} \times S^{2}$.

Received by the editors December 29, 1975.

AMS (MOS) subject classifications (1970). Primary 57E25.

Key words and phrases. Group action, 4-manifold, orbit space, equivariant homeomorphism, equivariant plumbing, quadratic form.

๑ American Mathematical Society 1977 
2. Preliminaries. In this section we fix the notation and terminology which we shall use. Recall that an action of a compact Lie group $G$ on a manifold $N$ is called locally smooth if each $x \in N$ has a slice which is a disk on which the action of the isotropy group $G_{x}$ is equivalent to an orthogonal action. All group actions which we discuss are assumed to be locally smooth and effective. See [1] for further terminology.

Throughout this paper $M$ will denote a simply connected oriented 4-manifold with an action of the circle group $S^{1}$. For any subset $X$ of $M, X^{*}$ denotes its image in the orbit space $M^{*}$ and $p: M \rightarrow M^{*}$ is the orbit map. Furthermore, if we are given a set $X^{*}$ in $M^{*}$, we let $X=p^{-1}\left(X^{*}\right)$ when no confusion is caused by this notation. We let $F$ be the fixed point set of $M, E$ the union of the exceptional orbits, and $P$ the union of the principal orbits.

All homology and cohomology used in this paper has $\mathbf{Z}$ coefficients. We shall of ten allow ourselves to confuse a loop in $M$ with the element in $\pi_{1}(M)$ or $H_{1}(M)$ which the loop represents.

The Orlik-Raymond-Seifert classification of $S^{1}$-actions on 3-manifolds [9], [11] will be needed in $\S \S 3$ and 5. We outline the aspects of the theory which will be of import there and refer the reader to [9] for further details. An $S^{1}$-action on a closed oriented 3-manifold $W$ is determined up to orientation-preserving equivariant homeomorphism by a collection of invariants $\left\{b ;(o, g, \bar{h}, 0) ;\left(\alpha_{1}, \beta_{1}\right), \ldots,\left(\alpha_{n}, \beta_{n}\right)\right\}$ as follows. The orbit space $W^{*}$ is a surface of genus $g$ with $h$ boundary components each corresponding to a circle of fixed points in $W$, and $W^{*}$ is oriented so that followed by the natural orientation on the orbits the orientation of $W$ is obtained. There are $n$ exceptional orbits each of which is assigned a pair of integers $\left(\alpha_{i}, \beta_{i}\right)$ called Seifert invariants.

If $E^{*}=\left\{x_{1}^{*}, \ldots, x_{n}^{*}\right\}$ choose disjoint closed 2-disk neighborhoods $V_{i}^{*}$ of the $x_{i}^{*}$. If $x_{i} \in p^{-1}\left(x_{i}^{*}\right)$ there is a closed 2-disk slice $S_{i}$ at $x_{i}$ such that $S_{i}^{*}=V_{i}^{*}$. Orient $S_{i}$ so that its intersection number with the oriented orbit $p^{-1}\left(x_{i}^{*}\right)$ is +1 in the solid torus $V_{i}$. On $\partial V_{i}$ let $m_{i}$ be an oriented boundary curve of $S_{i}$ and let $h_{i}$ be an oriented orbit. If the isotropy group at $x_{i}$ is $\mathbf{Z}_{\alpha_{i}}$ an oriented section $q_{i}$ of the action on $\partial V_{i}$ is specified up to homology by the homology relation $m_{i} \sim \alpha_{i} q_{i}+\beta_{i} h_{i}$ where $\alpha_{i}$ and $\beta_{i}$ are relatively prime and $0<\beta_{i}<\alpha_{i}$. The Seifert invariants $\left(\alpha_{i}, \beta_{i}\right)$ determine $V_{i}$ up to orientation-preserving equivariant homeomorphism. If the orientation on $V_{i}$ is reversed, the Seifert invariants become $\left(\alpha_{i}, \alpha_{i}-\beta_{i}\right)$ and if $q_{i}^{\prime}$ is the new section, $q_{i}^{\prime} \sim-q_{i}$ - $h_{i}$. The action of the isotropy group $\mathbf{Z}_{\alpha_{i}}$ on $S_{i}$ is orientation-preserving equivariantly homeomorphic to an action of $\mathbf{Z}_{\alpha_{i}}$ on $D^{2}$ :

$$
\frac{2 \pi}{\alpha_{i}} \times(r, \theta) \rightarrow\left(r, \theta+\frac{2 \pi v_{i}}{\alpha_{i}}\right) .
$$


The pair $\left[\alpha_{i}, \nu_{i}\right]$ are called the orbit invariants of $p^{-1}\left(x_{i}^{*}\right)$ and satisfy $\beta_{i} \nu_{i} \equiv 1$ $\left(\bmod \alpha_{i}\right)$.

The integer $b$ is the obstruction to extending the section $q_{1} \cup \cdots \cup q_{n}$ over all of $W^{*}-\cup \dot{V}_{i}^{*}$. So if $\bar{h} \neq 0$ we have $b=0$. If $\bar{h}=0$ let $V_{0}^{*}$ be a 2-disk in $W^{*}$ disjoint from $\cup_{i=1}^{n} V_{i}^{*}$. If $m_{0}$ is an oriented meridian on $\partial V_{0}$ and $q$ is the restriction to $\partial V_{0}$ of the oriented section $q_{1} \cup \cdots \cup q_{n}$ extended over $W^{*}-\cup_{i=0}^{n} \dot{V}_{i}^{*}$ then $q \sim-m_{0}+b h$, where $h$ is an oriented orbit. Reversing the orientation of the action on $W$ changes the invariants to $\left\{-n-b ;(o, g, \bar{h}, 0) ;\left(\alpha_{1}, \alpha_{1}-\beta_{1}\right), \ldots,\left(\alpha_{n}, \alpha_{n}-\beta_{n}\right)\right\}$. Finally, we note that the free action $\{b ;(o, 0,0,0)\}$ is the principal $S^{1}$-bundle over $S^{2}$ with Euler number $-b$ (see $[8$, p. 25]). $M^{4}$.

3. The weighted orbit space. In this section we describe the orbit structure of

Proposition (3.1). (a) The orbit space $M^{*}$ is a simply connected 3-manifold with $\partial M^{*} \subset F^{*}$.

(b) The set $F^{*}-\partial M^{*}$ is finite, and $F^{*}$ is nonempty.

(c) The closure of $E^{*}$ is a collection of polyhedral arcs and simple closed curves in $M^{*}$. The components of $E^{*}$ are open arcs on which orbit types are constant, and these arcs have closures with distinct endpoints in $F^{*}-\partial M^{*}$.

PROOF. If $x \in E$ the isotropy group at $x$ is a finite cyclic group which acts as a group of rotations on some 3-disk slice $S_{x}$ at $x$ with axis of rotation $E \cap S_{x}$. Then $S_{x}^{*}$ is a neighborhood of $x^{*}$ and the pair $\left(S_{x}^{*}, E^{*} \cap S_{x}^{*}\right)$ is homeomorphic to $\left(D^{2} \times I, 0 \times I\right)$.

If $y \in F$ there is a closed 4-disk slice $S_{y}$ at $y$ on which the $S^{1}$-action is the cone at $y$ of the action on the boundary 3 -sphere $\partial S_{y}$. The only $S^{1}$-actions on $S^{3}$ are of the form

$$
\begin{gathered}
\{ \pm 1 ;(o, 0,0,0)\}, \quad\{0 ;(o, 0,1,0)\}, \quad\{b ;(o, 0,0,0) ;(\alpha, \beta)\} \quad \text { and } \\
\left\{-1 ;(o, 0,0,0) ;(\alpha, \beta),\left(\alpha^{\prime}, \beta^{\prime}\right)\right\} .
\end{gathered}
$$

For these various cases we have $\left(S_{y}^{*}, E^{*} \cup F^{*}, F^{*}\right)$ homeomorphic to $\left(D^{3}, 0\right.$, $0),\left(D^{2} \times I, D^{2} \times 0, D^{2} \times 0\right),\left(D^{2} \times[-1,1], 0 \times[0,1],(0,0)\right)$, and $\left(D^{2} \times\right.$ $[-1,1], 0 \times[-1,1],(0,0))$ respectively. In the last case $\alpha \neq \alpha^{\prime}$.

It is well known that $M^{*}$ is simply connected. Since an $x^{*} \in P^{*}$ certainly has a 3-disk neighborhood in $M^{*}$, (a) is proved, and (b) follows from the formula $\chi(F)=\chi(M)=2+\operatorname{rank} H_{2}(M)$. A result of Montgomery and Yang [7, Lemma 2.3] implies that there is no simple closed curve in $E^{*}$ on which orbit types are constant. This is proved in [7] for the case $M=S^{4}$, but the same proof holds for any simply connected $M^{4}$. Thus $E^{*}$ consists of a collection of open arcs each of constant orbit type. Since $P$ is open in $M$, $E^{*} \cup F^{*}=M^{*}-P^{*}$ is compact and (c) follows. 
We remark that since $M^{*}$ is simply connected, it follows from duality that the components of $\partial M^{*}$ are 2-spheres.

(3.2) Since $M$ is oriented, an orientation on $M^{*}$ is determined, so that, followed by the natural orientation on the orbits, the orientation of $M$ is obtained. Given an oriented submanifold $X^{*}$ of $M^{*}$ we use the above convention to orient $X$. We say that a codimension one submanifold of an oriented manifold is oriented by some given normal to mean that the orientation on the submanifold followed by the normal gives the orientation of the ambient manifold. The boundary of an oriented manifold is to be oriented by the inward normal unless it is explicitly stated to the contrary.

Now suppose $X^{*} \subset E^{*} \cup F^{*}$. We shall reserve the term "regular neighborhood" of $X^{*}$ for those regular neighborhoods $N^{*}$ of $X^{*}$ which satisfy $N^{*} \cap\left(E^{*} \cup F^{*}\right)=X^{*}$.

(3.3) We assign to $M^{*}$ the following orbit data.

(a) For each boundary component $F_{i}^{*}$ of $M^{*}$ choose a regular neighborhood $F_{i}^{*} \times[0,1]$ and orient $F_{i}^{*} \times 1$ by the normal out of $F_{i}^{*} \times[0,1]$. The restriction of the orbit map gives a principal $S^{1}$-bundle over $F_{i}^{*} \times 1$ and we assign to $F_{i}^{*}$ the Euler number of this bundle [5]. This is clearly independent of our choice of collar. We shall call $F_{i}^{*}$ a weighted 2-sphere.

(b) If $x^{*} \in F^{*}-\left(\partial M^{*} \cup \mathrm{cl} E^{*}\right)$ let $B^{*}$ be a polyhedral 3-disk neighborhood of $x^{*}$ with $B^{*}-x^{*} \subset P^{*}$. Restriction of the orbit map gives a principal $S^{1}$-bundle over $\partial B^{*}$ with total space a 3 -sphere. Orient $\partial B^{*}$ by the normal out of $B^{*}$ and assign to $x^{*}$ the Euler number, \pm 1 , of the bundle.

(c) Suppose $L^{*}$ is a simple closed curve in $E^{*} \cup F^{*}$. To each component of $E^{*}$ in $L^{*}$ we assign Seifert invariants. First fix an orientation on $L^{*}$; this induces an orientation on each component $J^{*}$ of $E^{*}$ in $L^{*}$. Let $y^{*}$ be an endpoint of $\mathrm{cl} J^{*}$ and let $B^{*}$ be a polyhedral 3-disk neighborhood of $y^{*}$ such that $B^{*} \cap\left(E^{*} \cup F^{*}\right)=B^{*} \cap L^{*}$ is an arc and $B^{*} \cap F^{*}=y^{*}$. If $\partial B^{*}$ is oriented by the normal with direction $J^{*}$ then $\partial B$ is an oriented 3-sphere. Assign to $J^{*}$ the Seifert invariants $(\alpha, \beta)$ of the orbit in $\partial B$ with image in $J^{*}$. The covering homotopy theorem of Palais [1] implies that this definition is independent of the choices made.

The weights assigned to $L^{*}$ consist of the oriantation and the Seifert invariants. We abbreviate this system of weights by $\left\{\left(\alpha_{1}, \beta_{1}\right), \ldots,\left(\alpha_{n}, \beta_{n}\right)\right\}$ where the order of the $\left(\alpha_{i}, \beta_{i}\right)$ is determined up to a cyclic permuation, and we call $L^{*}$ a weighted circle. If the orientation of $L^{*}$ is reversed each $\left(\alpha_{i}, \beta_{i}\right)$ becomes $\left(\alpha_{i}, \alpha_{i}-\beta_{i}\right)$ and we regard the resulting weighted circle as equivalent to the first.

(d) If $A^{*}$ is an arc which is a component of $E^{*} \cup F^{*}$, we orient $A^{*}$ and assign Seifert invariants as in (c). If $y^{*}$ is the initial or final point of $A^{*}$ and $B^{*}$ is a small 3-disk neighborhood of $y^{*}$, then proceeding as in (c) $\partial B$ has the 
$S^{1}$-action $\{b ;(o, 0,0,0) ;(\alpha, \beta)\}$. Assign this integer $b$ to $y^{*}$. We call $A^{*}$ a weighted arc and write the weight system as $\left[b^{\prime} ;\left(\alpha_{1}, \beta_{1}\right), \ldots,\left(\alpha_{n}, \beta_{n}\right) ; b^{\prime \prime}\right]$. Reversing the orientation on $A^{*}$ changes the weight system to [ $-1-b^{\prime \prime} ;\left(\alpha_{n}\right.$, $\left.\left.\alpha_{n}-\beta_{n}\right), \ldots,\left(\alpha_{1}, \alpha_{1}-\beta_{1}\right) ;-1-b^{\prime}\right]$ which we regard as equivalent to the original weight system on $A^{*}$.

The oriented orbit space $M^{*}$ together with the above collection of weights is called a weighted orbit space.

(3.4) An isomorphism of weighted orbit spaces $M_{1}^{*}$ and $M_{2}^{*}$ is an orientation-preserving homeomorphism which carries the weights of $M_{1}^{*}$ isomorphically onto the weights of $M_{2}^{*}$.

Lemma (3.5). (a) If $\left(\alpha_{i}, \beta_{i}\right)$ and $\left(\alpha_{i+1}, \beta_{i+1}\right)$ are the Seifert invariants assigned to adjacent arcs in some weighted arc or circle then

$$
\left|\begin{array}{cc}
\alpha_{i} & \beta_{i} \\
\alpha_{i+1} & \beta_{i+1}
\end{array}\right|= \pm 1 .
$$

(b) If $\left[b^{\prime} ;\left(\alpha_{1}, \beta_{1}\right), \ldots,\left(\alpha_{n}, \beta_{n}\right) ; b^{\prime \prime}\right]$ is a weighted arc then $b^{\prime} \alpha_{1}+\beta_{1}= \pm 1$ and $b^{\prime \prime} \alpha_{n}+\beta_{n}= \pm 1$. (So for $i=1$ or $n, \beta_{i}=1$ or $\alpha_{i}-1$, and $b^{\prime}$ and $b^{\prime \prime}$ can only take on the values 0 or -1 .)

Proof. (a) If $y^{*} \in F^{*}$ belongs to the closure of both arcs involved and if $B^{*}$ is a small polyhedral 3-disk containing $y^{*}$ then the action on $\partial B$ is $\{-1$; $\left.(o, 0,0,0) ;\left(\alpha_{i}, \beta_{i}\right),\left(\alpha_{i+1}, \alpha_{i+1}-\beta_{i+1}\right)\right\}$. The result now follows from [ 9 , Theorem 4]. Similar considerations prove (b).

Proposition (3.6). Let $W^{*}$ be a regular neighborhood in $M^{*}$ of a weighted arc or circle. The weights determine $W=p^{-1}\left(W^{*}\right)$ up to orientation-preserving equivariant homeomorphism.

PROof. We give the proof for the case of a weighted circle; the proof in the other case is similar. Let $K^{*}$ and $L^{*}$ be isomorphically weighted circles with weight systems $\left\{\left(\alpha_{1}, \beta_{1}\right), \ldots,\left(\alpha_{n}, \beta_{n}\right)\right\}$, and let $U^{*}$ and $W^{*}$ be the respective regular neighborhoods. By deforming an orientation-preserving homeomorphism we obtain an isomorphism $f^{*}: U^{*} \rightarrow W^{*}$.

In $U^{*}$ let $y_{i}^{*} \in F^{*}$ be the initial point of the arc with Seifert invariants $\left(\alpha_{i}\right.$, $\beta_{i}$ ) and choose $x_{i}^{*}$ in the interior of this arc. Let $I_{i}^{*}$ denote the interval $\left[x_{i-1}^{*}\right.$, $x_{i}^{*}$ ] of $K^{*}$. Representing $U^{*}$ as $D^{2} \times K^{*}$ we let $B_{i}^{*}=D^{2} \times I_{i}^{*}$. Using the covering homotopy theorem of Palais along with the fact that the action is locally smooth, one sees that the action on $B_{i}$ is orientation-preserving equivalent to the cone at $y_{i}$ of the action on $\partial B_{i}$, the equivalence being the identity on $\partial B_{i}$. Similarly the action on $p_{W}^{-1}\left(f^{*}\left(B_{i}^{*}\right)\right)$ is equivalent to the cone of the action on its boundary. 
Let $V_{i}^{*}=D^{2} \times x_{i}$ and $Y_{i}^{*}=f^{*}\left(V_{i}^{*}\right)$ be oriented by $K^{*}$ and $L^{*}$. Since $f^{*}$ is a weighted isomorphism there is an orientation-preserving equivariant homeomorphism $g_{1}: \cup V_{i} \rightarrow \cup Y_{i}$. The induced $g_{1}^{*}$ is also orientation-preserving so there is an orbit-type preserving isotopy of $g_{1}^{*}$ to $f^{*} \mid \cup V_{i}^{*}$. Lifting this isotopy equivariantly we may suppose that $g_{1}^{*}=f^{*}$.

Let $q_{i}$ be the canonical section on $\partial V_{i}$. It follows from the classification of $S^{1}$-actions on $S^{3}$ that on $\partial B_{i}-\operatorname{int}\left(V_{i-1} \cup V_{i}\right)$ we have $q_{i-1} \sim-q_{i}^{\prime}-h_{i}^{\prime}$ where $q_{i}^{\prime}$ is the section of $\partial V_{i}$ oriented as a submanifold of $\partial B_{i}$. Thus $q_{i-1} \sim-q_{i}^{\prime}-h_{i}^{\prime} \sim q_{i}$, so the section $q_{i-1} \cup q_{i}$ extends to a section on $\partial B_{i}-\operatorname{int}\left(V_{i-1} \cup V_{i}\right)$. Hence $q_{1} \cup \cdots \cup q_{n}$ extends to a section over $\partial U^{*}$. Since $g_{1}\left(q_{i}\right)$ is homologous to the canonical section over $\partial Y_{i}$, also $g_{1}\left(q_{1}\right)$ $\cup \cdots \cup g_{1}\left(q_{n}\right)$ extends to a section over $\partial W^{*}$. Using these sections $g_{1}$ extends to an orientation-preserving equivariant homeomorphism $\partial U \cup \cup V_{i}$ $\rightarrow \partial W \cup \cup Y_{i}$. We now obtain the desired equivalence $g: U \rightarrow W$ via the cone structure.

(3.7). Addendum. The equivalence $g$ is constructed so that $g^{*} \mid \partial U^{*}=$ $f^{*} \mid \partial U^{*}$.

COROLLARY (3.8). If $L^{*}$ is a weighted circle in $M^{*}$ with regular neighborhood $W^{*}$ then $\partial W \cong T^{3}$ with the free $S^{1}$-action $\{0 ;(o, 1,0,0)\}$.

Proof. The orbit map $p: \partial W \rightarrow \partial W^{*} \cong T^{2}$ is a principal $S^{1}$-bundle which, according to the proof of (3.6), admits a cross-section.

Proposition (3.9). If $A^{*}=\left[b^{\prime} ;\left(\alpha_{1}, \beta_{1}\right), \ldots,\left(\alpha_{n}, \beta_{n}\right) ; b^{\prime \prime}\right]$ is a weighted arc in $M^{*}$ with regular neighborhood $W^{*}$, the $S^{1}$-action restricted to $\partial W$ is $\left\{b^{\prime \prime}-b^{\prime} ;(o, 0,0,0)\right\}$.

Proof. Let $F^{*}=\left\{y_{1}^{*}, \ldots, y_{n+1}^{*}\right\}$ and write $W^{*}=\bigcup B_{i}^{*}$ where $B_{i}^{*}$ is a polyhedral 3-disk containing $y_{i}^{*}, B_{i}^{*} \cap\left(E^{*} \cup F^{*}\right)$ is an arc containing no fixed point other than $y_{i}^{*}$, and $B_{i}^{*} \cap B_{i+1}^{*}=\partial B_{i}^{*} \cap \partial B_{i+1}^{*}$ is a 2-disk meeting $A^{*}$ at a single point.

Letting $q_{i}$ be the section on $\partial\left(B_{i} \cap B_{i+1}\right)$ as in (3.6) we have $q_{i} \sim q_{i+1}$ for $i=1, \ldots, n$. On $\operatorname{cl}\left(\partial B_{1}-B_{2}\right)$ let $m_{0}$ be an oriented meridional curve. Since the $S^{1}$-action on $\partial B_{1}$ is $\left\{-b^{\prime}-1 ;(0,0,0,0) ;\left(\alpha_{1}, \alpha_{1}-\beta_{1}\right)\right\}$ we have $m_{0} \sim-q_{1}^{\prime}-\left(b^{\prime}+1\right) h_{1}^{\prime} \sim q_{1}-b^{\prime} h_{1} \sim q_{2}-b^{\prime} h_{2} \sim \cdots \sim q_{n}-b^{\prime} h_{n}$. The action on $\partial B_{n+1}$ is $\left\{b^{\prime \prime} ;(o, 0,0,0) ;\left(\alpha_{n}, \beta_{n}\right)\right\}$ so $q_{n} \sim-m_{n+1}^{\prime}+b^{\prime \prime} h_{n+1}^{\prime}$. We have $m_{0} \sim-m_{n+1}^{\prime}+\left(b^{\prime \prime}-b^{\prime}\right) h_{n+1}^{\prime}$. So the action on $\partial W$ is as claimed.

(3.10) Since $\{b ;(o, 0,0,0)\} \cong L(b, 1)$, if $b^{\prime}=b^{\prime \prime}$ in (3.9) then $\partial W \cong$ $L(0,1)=S^{2} \times S^{1}$. It is easily seen using (3.5)(a) that this must be the case unless some $\left(\alpha_{i}, \beta_{i}\right)=(2,1)$. If $b^{\prime} \neq b^{\prime \prime}$ then $\partial W \cong L( \pm 1,1)=S^{3}$.

4. Equivariant plumbing. The equivariant plumbing of 2-disk bundles over $S^{2}$ is used in this section to form building blocks which will be used in $\S 5$ to 
construct manifolds with $S^{1}$-actions and in $\$ 7$ to construct manifolds with $T^{2}$-actions. We quickly describe our notation which is borrowed from [8]. See also [5] and [2].

(4.1) Write $S^{2}=B_{1} \cup B_{2}$ as the union of its upper and lower hemispheres. Coordinatize $B_{i} \times D_{i}^{2}(i=1,2)$ using polar coordinates on $B_{i}$ and $D_{i}$. For relatively prime integers $u_{i}$ and $v_{i}$ define an $S^{1}$-action by $S^{1} \times B_{i} \times D_{i} \rightarrow B_{i}$ $\times D_{i}, \phi \times(r, \gamma, s, \delta) \rightarrow\left(r, \gamma+u_{i} \phi, s, \delta+v_{i} \phi\right)$. Then if $u_{2}=-u_{1}$ and $v_{2}=-\omega u_{1}+v_{1}$ we obtain $Y_{\omega}=B_{1} \times D_{1} \cup_{G} B_{2} \times D_{2}$ by means of the equivariant pasting $G: \partial B_{1} \times D_{1} \rightarrow \partial B_{2} \times D_{2}, G(1, \gamma, s, \delta)=(1,-\gamma, s$, $-\omega \gamma+\delta)$. The resulting manifold $Y_{\omega}$ is the $D^{2}$-bundle over $S^{2}$ with Euler number $\omega$; i.e. $\omega$ is the self-intersection number of the zero section of $Y_{\omega}$.

Given $Y_{\omega_{1}}$ and $Y_{\omega_{2}}$ with $u_{2,1}=v_{1,2}$ and $v_{2,1}=u_{1,2}$ (or $u_{2,1}=-v_{1,2}$ and $v_{2,1}=-u_{1,2}$ ) we may equivariantly plumb together $Y_{\omega_{1}}$ and $Y_{\omega_{2}}$ with sign +1 (sign - 1) by identifying $B_{2,1} \times D_{2,1}$ with $B_{1,2} \times D_{1,2}$ by means of the equivariant homeomorphism $(r, \gamma, s, \delta) \rightarrow(s, \delta, r, \gamma)((r, \gamma, s, \delta) \rightarrow(s,-\delta, r$, $-\gamma)$ ). The resulting manifold $Y_{\omega_{1}} \square Y_{\omega_{2}}$ then has an induced $S^{1}$-action.

Similarly one may define (effective) $T^{2}$-actions on $Y_{\omega}$ by means of integers $u_{i}, v_{i}, w_{i}$, and $t_{i}$, where

$$
\left|\begin{array}{cc}
u_{i} & w_{i} \\
v_{i} & t_{i}
\end{array}\right|= \pm 1
$$

The corresponding $T^{2}$-action on $B_{i} \times D_{i}$ is given by $(\phi, \theta) \times(r, \gamma, s, \delta) \rightarrow(r$, $\left.\gamma+u_{i} \phi+w_{i} \theta, s, \delta+v_{i} \phi+t_{i} \theta\right)$. The pasting $G$ defined above will be $T^{2}$ equivariant if also $w_{2}=-w_{1}$ and $t_{2}=-\omega w_{1}+t_{1}$. The plumbing $Y_{\omega_{1}} \square Y_{\omega_{2}}$, say with sign +1 , may then be constructed $T^{2}$-equivariantly if also $w_{2,1}=t_{1,2}$ and $t_{2,1}=w_{1,2}$.

(4.2) For the $S^{1}$-action on $B_{i} \times D_{i}$ described in (4.1), suppose neither $u_{i}$ nor $v_{i}$ is 0 . The orbit space $\left(B_{i} \times D_{i}\right)^{*}$ of the action may be viewed as the suspension of $D^{2}$ in $\mathbf{R}^{3}$, i.e. as $\{(\rho, \theta, z)|z \in[-1,1], 0 \leqslant \rho \leqslant 1-| z \mid\}$, with orbit map $p(r, \gamma, s, \delta)=(r s, u \delta-v \gamma, r-s)$. The orientation induced on $\left(B_{i} \times D_{i}\right)^{*}$ from the action is just the orientation $\left(B_{i} \times D_{i}\right)^{*}$ inherits as a submanifold of $\mathbf{R}^{3}$.

Following (3.3) we assign weights to the arc $0 \times[-1,1]$ in $\left(B_{i} \times D_{i}\right)^{*}$. It will be convenient here to use orbit invariants rather than Seifert invariants. Orient the arc from -1 to +1 . To compute the invariants on $0 \times(0,1]$ use the outward normal to orient $\partial\left(B_{i} \times D_{i}\right)^{*}$. This orientation lifts to the orientation by the inward normal on $\partial\left(B_{i} \times D_{i}\right)$, and then the slice (1, $0) \times D_{i}$ at $(1,0,0,0)$ has orientation $\left(\operatorname{sgn} u_{i}\right)$ times the usual orientation on $D_{i}$ (so as to have intersection number +1 with an oriented orbit). It now follows from the definition that the orbit invariants are $\left[\left|u_{i}\right|,\left(\operatorname{sgn} u_{i}\right) v_{i}\right]$. Similarly the orbit invariants assigned to $0 \times[-1,0)$ are $\left[\left|v_{i}\right|,-\left(\operatorname{sgn} v_{i}\right) u_{i}\right]$. Note that if 
$u_{i}>2$ or $v_{i}>2$ then changing the sign on exactly one of the $u_{i}$ or $v_{i}$ gives the opposite orbit invariants.

If instead of $p$ we view the map $p^{\prime}(r, \gamma, s, \delta)=(r s, v \gamma-u \delta, r-s)$ as the orbit map, then the weighted orbit space $\left(B \times D_{i}\right)^{*}$ is the same as that given above except that it has the opposite orientation.

(4.3) We now catalogue some fundamental $S^{1}$ and $T^{2}$-actions on the disk bundles $Y_{\omega}$. According to (4.1) $S^{1}$ and $T^{2}$-actions on $Y_{\omega}$ are described by giving the matrix

$$
\left(\begin{array}{llll}
u_{1} & u_{2} & w_{1} & w_{2} \\
v_{1} & v_{2} & t_{1} & t_{2}
\end{array}\right)
$$

which satisfies certain conditions. The weighted orbit space $Y^{*}$ of the $S^{1}$. action may be computed using (4.2) and the descriptions in $\$ 2$. Throughout, $\varepsilon= \pm 1, n$ is an arbitrary integer, and pairs $(\alpha, \beta)$ consist of relatively prime integers $0<\beta<\alpha$.

(a) Suppose

$$
\varepsilon^{\prime}=\left|\begin{array}{ll}
\alpha^{\prime} & \beta^{\prime} \\
\alpha & \beta
\end{array}\right|= \pm 1, \quad \varepsilon^{\prime \prime}=\left|\begin{array}{ll}
\alpha & \beta \\
\alpha^{\prime \prime} & \beta^{\prime \prime}
\end{array}\right|= \pm 1,
$$

and

$$
\omega=\varepsilon^{\prime} \varepsilon^{\prime \prime}\left|\begin{array}{ll}
\alpha^{\prime} & \beta^{\prime} \\
\alpha^{\prime \prime} & \beta^{\prime \prime}
\end{array}\right|
$$

Then

$$
\left(\begin{array}{llll}
\varepsilon \alpha & -\varepsilon \alpha & \varepsilon(b+n \alpha) & -\varepsilon(\beta+n \alpha) \\
\varepsilon \varepsilon^{\prime} \alpha^{\prime} & -\varepsilon \varepsilon^{\prime \prime} \alpha^{\prime \prime} & \varepsilon \varepsilon^{\prime}\left(\beta^{\prime}+n \alpha^{\prime}\right) & -\varepsilon \varepsilon^{\prime \prime}\left(\beta^{\prime \prime}+n \alpha^{\prime \prime}\right)
\end{array}\right)
$$

describes actions on $Y_{\omega}$ with $Y_{\omega}^{*} \cong D^{3}$ :

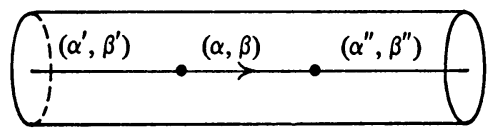

(b) Suppose

$$
\begin{aligned}
\varepsilon^{\prime \prime}=\left|\begin{array}{ll}
\alpha & \beta \\
\alpha^{\prime \prime} & \beta^{\prime \prime}
\end{array}\right| & = \pm 1, \quad b \alpha+\beta= \pm 1, \\
\varepsilon^{\prime} & =\left|\begin{array}{cc}
1 & |b| \\
\alpha & \beta
\end{array}\right|,
\end{aligned}
$$

and

$$
\omega=\varepsilon^{\prime} \varepsilon^{\prime \prime}\left|\begin{array}{cc}
1 & |b| \\
\alpha^{\prime \prime} & \beta^{\prime \prime}
\end{array}\right| .
$$


Then

$$
\left(\begin{array}{llll}
\varepsilon \alpha & -\varepsilon \alpha & \varepsilon(\beta+n \alpha) & -\varepsilon(\beta+n \alpha) \\
\varepsilon \varepsilon^{\prime} & -\varepsilon \varepsilon^{\prime \prime} \alpha^{\prime \prime} & \varepsilon \varepsilon^{\prime}(|b|+n) & -\varepsilon \varepsilon^{\prime \prime}\left(\beta^{\prime \prime}+n \alpha^{\prime \prime}\right)
\end{array}\right)
$$

describes actions on $Y_{\omega}$ with $Y_{\omega}^{*} \cong D^{3}$ :

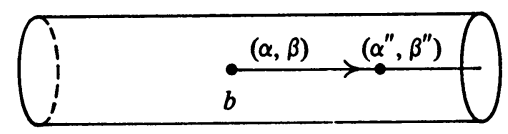

(c) If

$$
\begin{aligned}
b^{\prime} \alpha+\beta & = \pm 1, \quad b^{\prime \prime} \alpha+\beta= \pm 1, \quad \varepsilon^{\prime}=\left|\begin{array}{cc}
1 & \left|b^{\prime}\right| \\
\alpha & \beta
\end{array}\right| \\
\varepsilon^{\prime \prime} & =\left|\begin{array}{cc}
\alpha & \beta \\
1 & \left|b^{\prime \prime}\right|
\end{array}\right|, \quad \text { and } \omega=\varepsilon^{\prime} \varepsilon^{\prime \prime}\left|\begin{array}{cc}
1 & \left|b^{\prime}\right| \\
1 & \left|b^{\prime \prime}\right|
\end{array}\right|
\end{aligned}
$$

then

$$
\left(\begin{array}{cccc}
\varepsilon \alpha & -\varepsilon \alpha & \varepsilon(b+n \alpha) & -\varepsilon(b+n \alpha) \\
\varepsilon \varepsilon^{\prime} & -\varepsilon \varepsilon^{\prime \prime} & \varepsilon \varepsilon^{\prime}\left(\left|b^{\prime}\right|+n\right) & -\varepsilon \varepsilon^{\prime \prime}\left(\left|b^{\prime \prime}\right|+n\right)
\end{array}\right)
$$

defines actions on $Y_{\omega}$ with $Y_{\omega}^{*}=D^{3}$ :

$$
\underset{b^{\prime}}{\stackrel{(\alpha, \beta)}{\longrightarrow}} \stackrel{b^{\prime \prime}}{\longrightarrow}
$$

(d) Let $\varepsilon^{\prime}, \varepsilon^{\prime \prime}= \pm 1$ and $\omega=-\varepsilon^{\prime}-\varepsilon^{\prime \prime}$. Then

$$
\left(\begin{array}{llll}
\varepsilon & -\varepsilon & \varepsilon n & -\varepsilon n \\
-\varepsilon \varepsilon^{\prime} & \varepsilon \varepsilon^{\prime \prime} & -\varepsilon \varepsilon^{\prime}\left(n+\varepsilon^{\prime}\right) & \varepsilon \varepsilon^{\prime \prime}\left(n-\varepsilon^{\prime \prime}\right)
\end{array}\right)
$$

defines actions on $Y_{\omega} ; Y_{\omega}^{*} \cong D^{3}$ :

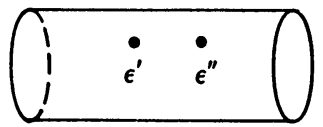

(e) If

$$
\begin{gathered}
b^{\prime \prime} \alpha^{\prime \prime}+\beta^{\prime \prime}= \pm 1, \quad \varepsilon^{\prime}= \pm 1, \\
\varepsilon^{\prime \prime}=\left|\begin{array}{cc}
1 & \left|b^{\prime \prime}\right| \\
\alpha^{\prime \prime} & \beta^{\prime \prime}
\end{array}\right|, \quad \text { and } \quad \omega=\varepsilon^{\prime \prime} \alpha^{\prime \prime}-\varepsilon^{\prime}
\end{gathered}
$$

then 


$$
\left(\begin{array}{llll}
\varepsilon & -\varepsilon & \varepsilon n & -\varepsilon n \\
-\varepsilon \varepsilon^{\prime} & -\varepsilon \varepsilon^{\prime \prime} \alpha^{\prime \prime} & -\varepsilon \varepsilon^{\prime}\left(n+\varepsilon^{\prime}\right) & -\varepsilon \varepsilon^{\prime \prime}\left(\beta^{\prime \prime}+\left(n-\left|b^{\prime \prime}\right|\right) \alpha^{\prime \prime}\right)
\end{array}\right)
$$

defines actions on $Y_{\omega}$ with $Y_{\omega}^{*} \cong D^{3}$ :

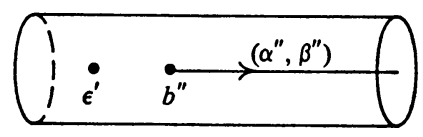

(f) Suppose

$$
\begin{aligned}
b^{\prime} \alpha^{\prime}+\beta^{\prime} & = \pm 1, \quad b^{\prime \prime} \alpha^{\prime \prime}+\beta^{\prime \prime}= \pm 1, \quad \varepsilon^{\prime}=\left|\begin{array}{cc}
\alpha^{\prime} & \beta^{\prime} \\
1 & \mid b^{\prime}
\end{array}\right| \\
\varepsilon^{\prime \prime} & =\left|\begin{array}{cc}
1 & \left|b^{\prime \prime}\right| \\
\alpha^{\prime \prime} & \beta^{\prime \prime}
\end{array}\right|, \quad \text { and } \omega=\varepsilon^{\prime} \alpha^{\prime}+\varepsilon^{\prime \prime} \alpha^{\prime \prime}
\end{aligned}
$$

Then

$$
\left(\begin{array}{llll}
\varepsilon & -\varepsilon & \varepsilon\left(\left|b^{\prime}\right|+n\right) & -\varepsilon\left(\left|b^{\prime}\right|+n\right) \\
\varepsilon \varepsilon^{\prime} \alpha^{\prime} & -\varepsilon \varepsilon^{\prime \prime} \alpha^{\prime \prime} & \varepsilon \varepsilon^{\prime}\left(\beta^{\prime}+n \alpha^{\prime}\right) & -\varepsilon \varepsilon^{\prime \prime}\left(\beta^{\prime \prime}+\left(n+\left|b^{\prime}\right|-\left|b^{\prime \prime}\right|\right) \alpha^{\prime \prime}\right)
\end{array}\right)
$$

defines actions on $Y_{\omega}$ with $Y_{\omega}^{*} \cong D^{3}$ :

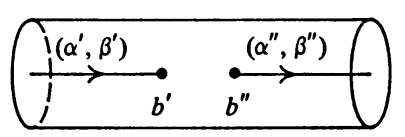

(g) Suppose

$$
b^{\prime} \alpha^{\prime}+\beta^{\prime}= \pm 1, \quad \varepsilon^{\prime}=\left|\begin{array}{cc}
\alpha^{\prime} & \beta^{\prime} \\
1 & \left|b^{\prime}\right|
\end{array}\right|, \quad \text { and } \quad \omega=\varepsilon^{\prime} \alpha^{\prime}
$$

Then

$$
\left(\begin{array}{llll}
\varepsilon & -\varepsilon & \varepsilon\left(\left|b^{\prime}\right|+n\right) & -\varepsilon\left(\left|b^{\prime}\right|+n\right) \\
\varepsilon \varepsilon^{\prime} \alpha^{\prime} & 0 & \varepsilon \varepsilon^{\prime}\left(\beta^{\prime}+n \alpha^{\prime}\right) & -\varepsilon
\end{array}\right)
$$

defines actions on $Y_{\omega}$, and $Y_{\omega}^{*}=D^{3}$ :

$$
\stackrel{i\left(\alpha^{\prime}, \beta^{\prime}\right)}{\longrightarrow} \underset{i}{b^{\prime}}
$$

(h) Let $\varepsilon^{\prime}= \pm 1$ and $\omega=-\varepsilon^{\prime}$. Then

$$
\left(\begin{array}{llll}
\varepsilon & -\varepsilon & \varepsilon & -\varepsilon n \\
-\varepsilon \varepsilon^{\prime} & 0 & -\varepsilon \varepsilon^{\prime}\left(n+\varepsilon^{\prime}\right) & -\varepsilon
\end{array}\right)
$$


describes actions on $Y_{\omega}$ with $Y_{\omega}^{*} \cong D^{3}$ :

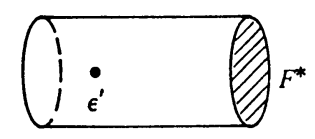

(i) The matrix

$$
\left(\begin{array}{cccc}
\varepsilon & -\varepsilon & n & -n \\
0 & 0 & \delta & \delta
\end{array}\right)
$$

for $\delta= \pm 1$ defines actions on $Y_{0}$ with $Y_{0}^{*} \cong D^{3}$ :

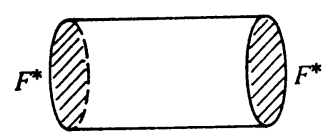

(j) For $\omega$ arbitrary and $\delta= \pm 1$ actions on $Y_{\omega}$ are defined by

$$
\left(\begin{array}{cccc}
0 & 0 & \delta & -\delta \\
\varepsilon & \varepsilon & n & -\omega \delta+n
\end{array}\right)
$$

and $Y_{\omega}^{*} \cong S^{2} \times I$ with $E^{*} \cup F^{*}=F^{*}=S^{2} \times 0$ with weight $\omega$.

LeMma (4.4). Let $L^{*}$ be the weighted circle $\left\{\left(\alpha_{1}, \beta_{1}\right), \ldots,\left(\alpha_{n}, \beta_{n}\right)\right\}$ in $M^{*}$, and for $i=1, \ldots, n$ let

$$
\omega_{i}=\left|\begin{array}{ll}
\alpha_{i-1} & \beta_{i-1} \\
\alpha_{i} & \beta_{i}
\end{array}\right|\left|\begin{array}{ll}
\alpha_{i} & \beta_{i} \\
\alpha_{i+1} & \beta_{i+1}
\end{array}\right|\left|\begin{array}{ll}
\alpha_{i-1} & \beta_{i-1} \\
\alpha_{i+1} & \beta_{i+1}
\end{array}\right|
$$

(identifying $-1 \equiv n$ and $n+1 \equiv 0$ ). Then an equivariant plumbing according to the graph

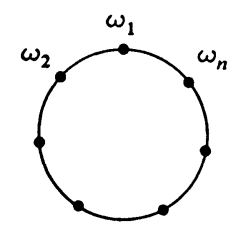

yields a 4-manifold $W$ with $S^{1}$-action and weighted orbit space $D^{2} \times S^{1}$ and $E^{*} \cup F^{*}=0 \times S^{1}$ isomorphic to $L^{*}$. Furthermore, this $S^{1}$-action extends to a $T^{2}$-action. Each plumbing, except perhaps for the last, $Y_{\omega_{n}} \square Y_{\omega_{1}}$ may be chosen to have sign +1 .

Proof. This follows immediately from (4.3)(a). Note that in this instance we may always choose $n=0$ in (4.3)(a); so all the plumbings may be constructed $T^{2}$-equivariantly.

LEMMA (4.5). For the $W$ constructed in (4.4), $E \cup F$ is a strong deformation retract of $W$. 
Proof. It is a general fact that the resultant manifold of a plumbing strong deformation retracts onto the union of the zero sections. See [2, V.2.2.].

(4.6) Let $S_{1}^{*}, \ldots, S_{t}^{*}$ be the collection of all the weighted sets of $M^{*}$ other than the weighted circles. If there are any weighted boundary components of $M^{*}$ we list these at the end. For each $i=1, \ldots, t-1$ let $\gamma_{i}^{*}$ be an arc in $M^{*}$ running from $S_{i}^{*}$ to $S_{i+1}^{*}$ such that the interior of the arc lies in $P^{*}$ and such that if $S_{i}^{*}$ is a weighted arc, $\gamma_{i}^{*}$ begins at the endpoint of $S_{i}^{*}$, and if $S_{i+1}^{*}$ is a weighted arc, $\gamma_{i}^{*}$ ends at the initial point of $S_{i+1}^{*}$.

Let $R^{*}$ be a regular neighborhood of $\cup S_{i}^{*} \cup \cup \gamma_{i}^{*}$. The following lemma is a direct result of the constructions in (4.3).

LEMMA (4.7). By means of an equivariant linear plumbing (with each plumbing of sign +1$)$ there may be constructed a 4-manifold $W$ with $S^{1}$-action and weighted orbit space isomorphic to $R^{*}$. Furthermore, this action extends to a $T^{2}$-action on $W$.

5. Constructions. Let $N^{*}$ be a regular neighborhood of $E^{*} \cup F^{*}$ in $M^{*}$ and let $\hat{M}^{*}=\operatorname{cl}\left(M^{*}-N^{*}\right)$. For a boundary component $T_{i}^{*}$ of $\hat{M}^{*}$ denote by $e_{i}$ the Euler number of the principal $S^{1}$-bundle $T_{i} \rightarrow T_{i}^{*}$. If $T_{i}^{*}$ is the boundary of a regular neighborhood of a weighted sphere or point then $e_{i}$ is just that weight. Corollary (3.8) implies that each $T_{i}^{*}$ bounding a regular neighborhood of a weighted circle has $e_{i}=0$. Finally, if $T_{i}^{*}$ bounds a regular neighborhood of a weighted arc $\left[b^{\prime} ;\left(\alpha_{1}, \beta_{1}\right), \ldots,\left(\alpha_{n}, \beta_{n}\right) ; b^{\prime \prime}\right]$ it follows from (3.9) that the $S^{1}$-action on $T_{i}$ is $\left\{b^{\prime}-b^{\prime \prime} ;(o, 0,0,0)\right\}$; so $e_{i}=b^{\prime \prime}-b^{\prime}$.

LEMMA (5.1). $\sum e_{i}=0$.

Proof. Let $\xi \in H^{2}\left(\hat{M}^{*}\right)$ be the Euler class of the principal $S^{1}$-bundle $\hat{M} \rightarrow \hat{M}^{*}$, and let $\mu \in H^{3}\left(\hat{M}^{*}, \partial \hat{M}^{*}\right)$ and $\mu_{i} \in H^{2}\left(T_{i}^{*}\right)$ be the generators corresponding to the orientation. We have the exact sequence $H^{2}\left(\hat{M}^{*}\right)$ $\rightarrow^{\rho} H^{2}\left(\bigcup T_{i}^{*}\right) \rightarrow^{\delta} H^{3}\left(\hat{M}^{*}, \partial \hat{M}^{*}\right)$. Now $H^{2}\left(\cup T_{i}^{*}\right)=\bigoplus H^{2}\left(T_{i}^{*}\right)$ and $0=$ $\delta \rho(\xi)=\delta\left(e_{1} \mu_{1}, \ldots, e_{n} \mu_{n}\right)=\left(\sum e_{i}\right) \mu$; so $\sum e_{i}=0$.

(5.2) We define a legally weighted simply connected 3-manifold to be an oriented simply connected compact 3-manifold $X^{*}$ along with the data:

(a) an integer $a_{i}$ assigned to each boundary component of $X^{*}$,

(b) a finite collection of points in int $X^{*}$ with each assigned an integer $b_{i}= \pm 1$, and

(c) a collection of weighted arcs and circles in int $X^{*}$ as in (3.3) and satisfying the criteria of Lemma (3.5). To each weighted arc $A_{i}^{*}=\left[b^{\prime} ;\left(\alpha_{1}\right.\right.$, $\left.\left.\beta_{1}\right), \ldots,\left(\alpha_{n}, \beta_{n}\right) ; b^{\prime \prime}\right]$ the integer $c_{i}=b^{\prime \prime}-b^{\prime}$ is assigned.

Furthermore, at least one of the above collections must be nonempty, and we require $\Sigma a_{i}+\sum b_{i}+\sum c_{i}=0$. 
The definition of isomorphism carries over from the class of weighted orbit spaces to the class of legally weighted simply connected 3-manifolds. It follows from (3.1) and (5.1) that the weighted orbit space $M^{*}$ of an $S^{1}$-action on a simply connected $M^{4}$ is legally weighted.

Now fix a legally weighted simply connected $X^{*}$. We shall construct a simply connected 4-manifold $M$ with $S^{1}$-action such that $M^{*}$ is isomorphic to $X^{*}$.

Construction (5.3). In analogy with (4.6) connect all the weighted points, spheres, and arcs of $X^{*}$ with arcs and take a regular neighborhood $R^{*}$. If each boundary component of $X^{*}$ is capped off with a 3-disk then $X^{*}$ becomes a homotopy 3-sphere, and the union of $R^{*}$ with these disks is a 3-disk. Thus $X_{1}^{*}=\operatorname{cl}\left(X^{*}-R^{*}\right)$ is a homotopy 3-disk and $\partial X_{1}^{*}=\partial R^{*}-\partial X^{*}$ is a 2-sphere. Using Lemma (4.7) we obtain a 4-manifold $R$ with $S^{1}$-action and weighted orbit space which we identify with $R^{*}$. If $e$ is the Euler number of the principal $S^{1}$-bundle $\partial R \rightarrow \partial R^{*}-\partial X^{*}$ obtained by restricting the action, then the argument of (5.1) shows $e+\sum a_{i}+\sum b_{i}+\sum c_{i}=0$; so $e=0$ since $X^{*}$ is legally weighted. Thus $\partial R \rightarrow \partial R^{*}-\partial X^{*}$ is a trivial $S^{1}$-bundle.

Let $L_{1}^{*}, \ldots, L_{m}^{*}$ be the weighted circles of $X^{*}$ and let $Q_{i}^{*}$ be a regular neighborhood of $L_{i}^{*}$ in $X_{1}^{*}$. Let $X_{2}^{*}=\operatorname{cl}\left(X_{1}^{*}-\cup Q_{i}^{*}\right)$ and equip $X_{2}=X_{2}^{*} \times$ $S^{1}$ with the $S^{1}$-action by translation in the second factor and orbit map $\pi$ : $X_{2} \rightarrow X_{2}^{*}$. There is an orientation-reversing equivariant homeomorphism $\sigma$ : $\partial R \rightarrow \pi^{-1}\left(R^{*} \cap X_{2}^{*}\right)$ with $\sigma^{*}=\mathrm{id}$. It is easily seen that $\sigma$ is unique up to vertical equivariant isotopy, i.e. equivariant isotopy over the identity, so the 4-manifold $R \cup_{\sigma} X$ is well defined up to orientation-preserving equivariant homeomorphism.

We view $L_{1}^{*} \cup \cdots \cup L_{m}^{*}$ as a link in the simply connected 3-manifold $X_{1}^{*}$ and $\pi_{1}\left(X_{2}^{*}\right)$ as the group of this link. Let $s$ be the section $s: X_{2}^{*} \rightarrow X_{2}^{*} \times 0 \subset$ $X_{2}$. If $R^{*}$ is empty then $\pi_{1}\left(R \cup_{\sigma} X_{2}\right)=s_{\#} \pi_{1}\left(X_{2}^{*}\right) \times \pi_{1}\left(S^{1}\right)$. If $R^{*}$ is nonempty then it follows from Van Kampen's Theorem that $\pi_{1}\left(R \cup_{\sigma} X_{2}\right)=$ $s_{\#} \pi_{1}\left(X_{2}^{*}\right)$, for $R$ is the result of a linear plumbing and thus is simply connected [2, V.2.10] and $R \cap F \neq \varnothing$. In particular, if there are no weighted circles, $\pi_{1}\left(R \cup_{\sigma} X\right) \approx \pi_{1}\left(X_{1}^{*}\right)=1$.

Using Lemma (4.4) construct for each $L_{i}^{*}$ a 4-manifold $Q_{i}$ with $S^{1}$-action and weighted orbit space identified with $Q_{i}^{*}$. Let $p_{i}: Q_{i} \rightarrow Q_{i}^{*}$ be the orbit map. It follows from (3.8) that there is an orientation-reversing equivariant homeomorphism $\tau_{i}: \partial Q_{i} \rightarrow \pi^{-1}\left(\partial Q_{i}^{*}\right)$ with $\tau_{i}^{*}=\mathrm{id}$. The maps $\tau_{i}$ are not unique and a choice must be made here. Let $M$ be the 4-manifold obtained by pasting each $Q_{i}$ to $R \cup_{\sigma} X$ via $\tau_{i}$.

We now describe the possibilities. On each $\partial Q_{i}^{*}$ choose a meridional curve $m_{i}^{*}$ and a longitudinal curve $l_{i}^{*}\left(l_{i}^{*}\right.$ bounds in $\left.X_{1}^{*}-L_{i}^{*}\right)$ both passing through some point $z_{i}^{*}$. Orient $l_{i}^{*}$ and $m_{i}^{*}$ so that $l_{i}^{*}$ followed by $m_{i}^{*}$ gives the 
orientation of $\partial Q_{i}^{*}$ as a boundary component of $X_{2}^{*}$. Let $z_{i}^{\prime}=s\left(z_{i}^{*}\right)$ and $z_{i} \in \tau_{i}^{-1}\left(z_{i}^{\prime}\right)$. Then $\pi_{1}\left(\partial Q_{i}, z_{i}\right)=\left\langle q_{i}\right\rangle \times\left\langle l_{i}\right\rangle \times\left\langle h_{i}\right\rangle$ where $q_{i}$ is a section over $m_{i}^{*}, l_{i}$ is a section over $l_{i}^{*}$, and $h_{i}$ is an oriented orbit containing $z_{i}$. Also $\pi_{1}\left(\pi^{-1}\left(\partial Q_{i}^{*}\right), z_{i}^{\prime}\right)=\left\langle m_{i}^{\prime}\right\rangle \times\left\langle l_{i}^{\prime}\right\rangle \times\left\langle h_{i}^{\prime}\right\rangle$ where $m_{i}^{\prime}=s\left(m_{i}^{*}\right), l_{i}^{\prime}=s\left(l_{i}^{*}\right)$, and $h_{i}^{\prime}$ is the orbit through $z_{i}^{\prime}$. The attaching $\tau_{i}$ is determined up to vertical equivariant isotopy by the relations $\tau_{i_{*}}\left(h_{i}\right)=h_{i}^{\prime}, \tau_{i_{*}}\left(l_{i}\right)=l_{i}^{\prime} h_{i}^{\prime \prime}$, and $\tau_{i_{*}}\left(q_{i}\right)=$ $m_{i}^{\prime} h_{i}^{\prime s_{i}}$ for integers $r_{i}$ and $s_{i}$. Actually, $\tau_{i}$ is determined by the corresponding homology relations.

Next we show that $\pi_{1}(M)=1$. This has already been done in the case where $M=R \cup_{\sigma} X_{2}$. Recall that $\pi_{1}\left(R \cup_{\sigma} X_{2}\right)$ is generated by $s_{\#} \pi_{1}\left(X_{2}^{*}\right)$ and an oriented orbit, and by Lemma (4.5), $\pi_{1}\left(Q_{1}, z_{1}\right)=\left\langle k_{1}\right\rangle \approx \mathbf{Z}$. The inclusion induced $i_{\#}: \pi_{1}\left(\partial Q_{1}, z_{1}\right) \rightarrow \pi_{1}\left(Q_{1}, z_{1}\right)$ satisfies $i_{\#}\left(h_{1}\right)=1$ and $i_{\#}\left(l_{1}\right)=k_{1}$. Now $m_{1}^{*}$ bounds a disk in $Q_{1}^{*}$ which meets $L_{1}^{*}$ in one point, say in the open arc with weight $(\alpha, \beta)$. So $1=i_{\#}\left(q_{1}\right)^{\alpha} i_{\#}\left(h_{1}\right)^{\beta}=i_{\#}\left(q_{1}\right)^{\alpha}$. Since it has finite order in $\pi_{1}\left(Q_{1}, z_{1}\right), i_{\#}\left(q_{1}\right)=1$.

Van Kampen's Theorem yields the relations $h_{1}^{\prime}=h_{1}=1,1=q_{1}=m_{1}^{\prime} h_{1}^{\prime s_{1}}$ $=m_{1}^{\prime}$, and $k_{1}=l_{1}=l_{1}^{\prime} h_{1}^{\prime r_{1}}=l_{1}^{\prime} \in j_{\#} \pi_{1}\left(X_{2}^{*}, z_{1}^{*}\right)$ in $\pi_{1}\left(R \cup_{\sigma} X_{2} \cup_{\tau_{1}} Q_{1}, z_{1}\right)$ where $j$ is $s$ composed with the inclusion into $R \cup_{\sigma} X_{2} \cup_{\tau_{1}} Q_{1}$. Let $G_{1}$ be the subgroup of $\pi_{1}\left(X_{2}^{*}, z_{1}^{*}\right)$ generated by the meridians on $Q_{1}^{*}$. Since

$$
j_{\#}\left(m_{1}^{*}\right)=m_{1}^{\prime}=1 \text {, }
$$

it follows easily that $j_{\#}\left(G_{1}\right)=1$. Thus $\pi_{1}\left(R \cup_{\sigma} X_{2} \cup_{\tau_{1}} Q_{1}, z_{1}\right)$ is $\pi_{1}\left(X_{2}^{*}, z_{1}^{*}\right)$ modulo the normal closure of $G_{1}$.

The link group $\pi_{1}\left(X_{2}^{*}\right)$ is generated by the meridians lying on the $\partial Q_{i}^{*}$ and sewing in $Q_{j}^{*}$ kills those meridians lying on $\partial Q_{j}^{*}$; so we finally obtain $\pi_{1}(M)=1$. Thus $M$ is a simply connected 4-manifold with $S^{1}$-action and weighted orbit space isomorphic to $X^{*}$. In (5.6) we show that $M$ is independent of the choices involved in attaching the $Q_{i}$.

LEMMA (5.4). Given any integer $n$, there is an equivariant homeomorphism $\Phi$ : $Q_{i} \rightarrow Q_{i}$ with $\Phi^{*}=$ id such that $\Phi\left(q_{i}\right) \sim q_{i}$ and $\Phi\left(l_{i}\right) \sim l_{i} h_{i}^{n}$.

Proof. Identify $Q_{i}^{*}$ with $D^{2} \times L_{i}^{*}$ and in $L_{i}^{*}$ choose a closed interval $J^{*}$ which does not meet $F^{*}$. The covering homotopy theorem of Palais implies that there is an equivariant homeomorphism

$$
f: p_{i}^{-1}\left(D^{2} \times y^{*}\right) \times I \rightarrow p_{i}^{-1}\left(D^{2} \times J^{*}\right)
$$

with $f\left(p_{i}^{-1}\left(D^{2} \times y^{*}\right) \times 0\right)=p_{i}^{-1}\left(D^{2} \times y^{*}\right)$ where $y^{*}$ is the initial point of $J^{*}$. The desired homeomorphism is

$$
\Phi(x)= \begin{cases}x, & x \notin p_{i}^{-1}\left(D^{2} \times J^{*}\right), \\ (2 \pi n t) \cdot x, & x=f(v, t) . \quad \square\end{cases}
$$

Lemma (5.5). For $i, k=1, \ldots, m$ let $\varepsilon_{k i}$ be the linking number of $L_{k}^{*}$ and $L_{i}^{*}$ 
in $X_{1}^{*}$. If $f: \cup_{1}^{m} \partial Q_{i}^{*} \rightarrow S^{1}$ satisfies

$$
\operatorname{deg}\left(f l_{i}^{*}\right)=\sum_{k} \varepsilon_{k i} \operatorname{deg}\left(f \mid m_{k}^{*}\right)
$$

for each $i$ then $f$ may be extended to a map $X_{2}^{*} \rightarrow S^{1}$.

Proof. Let $\zeta \in H_{3}\left(X_{2}^{*}, \partial X_{2}^{*}\right)$ be the fundamental class and let $\lambda$ and $\mu$ be the inclusions $\cup \partial Q_{i}^{*} \rightarrow^{\lambda} \partial X_{2}^{*} \rightarrow^{\mu} X_{2}^{*}$. Since $\partial X_{1}^{*}$ is a 2 -sphere we have isomorphisms $s$ and $t$ such that

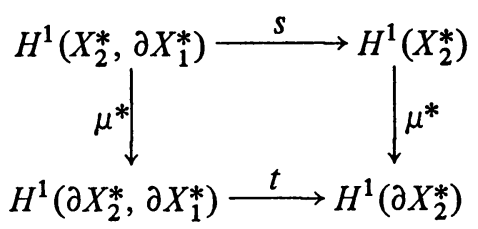

commutes.

The elements $m_{i}^{*}$ and $l_{i}^{*}$ form a basis for $H_{1}\left(\partial X_{2}^{*}\right)$. (We shall tacitly denote the isomorphism $\lambda_{*}$ by equality.) Let $\left\{m_{i}^{*}\right\}$ and $\left\{l_{i}^{*}\right\}$ denote the elements of the dual basis for $H^{1}\left(\partial X_{2}^{*}\right)$. Since $X_{2}^{*}$ is the complement of a regular neighborhood of the link $\cup L_{i}^{*}$ in the homotopy 3-disk $X_{1}^{*}, H_{1}\left(X_{2}^{*}\right)$ is free abelian with basis consisting of the $\mu_{*} m_{i}^{*}$. Let $\left[\mu_{*} m_{i}^{*}\right]$ denote the elements of the dual basis for $H^{1}\left(X_{2}^{*}\right)$.

By $[12,5.6 .20]$ we have the commutative diagram:

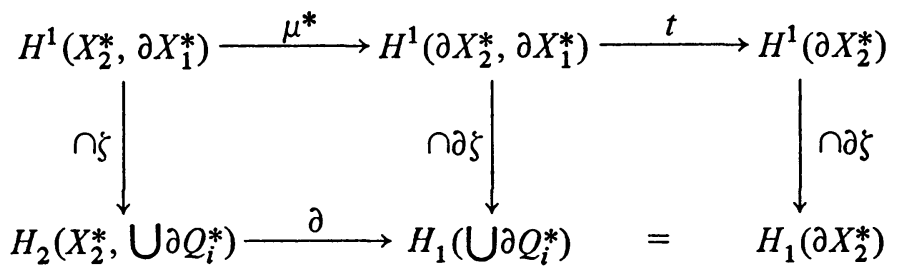

Thus $\partial\left(s^{-1}\left[\mu_{*} m_{i}^{*}\right] \cap \zeta\right)=t \mu^{*} s^{-1}\left[\mu_{*} m_{i}^{*}\right] \cap \partial \zeta=\mu^{*}\left[\mu_{*} m_{i}^{*}\right] \cap \partial \zeta$. Also we have $\left\{m_{i}^{*}\right\} \cap \partial \zeta=l_{i}^{*}$ and $\left\{l_{i}^{*}\right\} \cap \partial \zeta=-m_{i}^{*}$.

Each longitude $l_{i}^{*}$ spans a Seifert surface $S_{i}$ in $X_{i}^{*}$, and by general position we may suppose $S_{1} \cap Q_{j}^{*}$ consists of a finite number of disjoint 2-disks. It follows that in $H_{1}\left(X_{2}^{*}\right)$ we have $\mu_{*} l_{i}^{*}=\sum_{j} \varepsilon_{i j} \mu_{*} m_{j}^{*}$. The homomorphism $\mu^{*}$ is the transpose of $\mu_{*}$, so $\mu^{*}\left[\mu_{*} m_{i}^{*}\right]=\left\{m_{i}^{*}\right\}+\Sigma_{k} \varepsilon_{k i}\left\{l_{k}^{*}\right\}$. Thus $\mu^{*}\left[\mu_{*} m_{i}^{*}\right] \cap \partial \zeta$ $=l_{i}^{*}-\Sigma_{k} \varepsilon_{k i} m_{k}^{*}$.

The obstruction to extending $f$ is $\delta f^{*}(\iota) \in H^{2}\left(X_{2}^{*}, \partial Q_{i}^{*}\right)$ where $\iota$ is a generator of $H^{1}\left(S^{1}\right)$ [12, 8.1.12]. Computing the Kronecker index we have 


$$
\begin{aligned}
\left\langle\delta f^{*}(\iota), s^{-1}\left[\mu_{*} m_{i}^{*}\right] \cap \zeta\right\rangle=\left\langle f^{*}(\iota), \partial\left(s^{-1}\left[\mu_{*} m_{i}^{*}\right] \cap \zeta\right)\right\rangle \\
=\left\langle f^{*}(\iota), l_{i}^{*}-\sum_{k} \varepsilon_{k i} m_{k}^{*}\right\rangle=\left\langle\iota, f_{*}\left(l_{i}^{*}-\sum_{k} \varepsilon_{k i} m_{k}^{*}\right)\right\rangle \\
=\operatorname{deg}\left(f \mid l_{i}^{*}\right)-\sum_{k} \varepsilon_{k i} \operatorname{deg}\left(f \mid m_{k}^{*}\right),
\end{aligned}
$$

and the obstruction $\delta f^{*}(\iota)$ vanishes if and only if $\left\langle\delta f^{*}(\iota), s^{-1}\left[\mu_{*} m_{i}^{*}\right] \cap \zeta\right\rangle=0$ for each $i$, since the $s^{-1}\left[\mu_{*} m_{i}^{*}\right] \cap \zeta$ form a basis for $H_{2}\left(X_{2}^{*}, \cup \partial Q_{i}^{*}\right)$.

THEOREM (5.6). Construction (5.3) is independent of the choice of attaching maps.

Proof. Let $\tau_{1}$ and $\tau_{2}$ be equivariant embeddings: $\cup_{i=1}^{m} \partial Q_{i} \rightarrow \partial X_{2}$ with $\tau_{1}^{*}=\tau_{2}^{*}=\mathrm{id}$. Say that $\tau_{k}\left(l_{i}\right) \sim l_{i}^{\prime}+r_{i k} h_{i}^{\prime}$ and $\tau_{k}\left(q_{i}\right) \sim m_{i}^{\prime}+s_{i k} h_{i}^{\prime}$ for $k=1,2$. Choose a section $K^{\prime}$ over $\cup \partial Q_{i}^{*}$ oriented so that $K^{\prime} \cap p_{i}^{-1}\left(l_{i}^{*}\right) \sim l_{i}+a_{i} h_{i}$ and $K^{\prime} \cap p_{i}^{-1}\left(m_{i}^{*}\right) \sim q_{i}+b_{i} h_{i}$ for some integers $a_{i}$ and $b_{i}$. Using Lemma (5.4) choose an equivariant homeomorphism $\Psi: \cup Q_{i} \rightarrow \cup Q_{i}$ such that $\Psi^{*}=\mathrm{id}$ and for each $i$,

$$
\Psi\left(q_{i}\right) \sim q_{i} \text { and } \Psi\left(l_{i}\right) \sim l_{i}-\left[a_{i}+r_{i 1}-\sum_{j} \varepsilon_{j i}\left(b_{j}+s_{j 1}\right)\right] h_{i} .
$$

Then $K=\Psi\left(K^{\prime}\right)$ is a section over $\cup \partial Q_{i}^{*}$ with

$$
\tau_{1}(K) \cap \pi^{-1}\left(m_{i}^{*}\right) \sim m_{i}^{\prime}+\left(b_{i}+s_{i 1}\right) h_{i}^{\prime}
$$

and

$$
\tau_{1}(K) \cap \pi^{-1}\left(l_{i}^{*}\right) \sim l_{i}^{\prime}+\left(\sum_{j} \varepsilon_{j 1}\left(b_{j}+s_{j 1}\right)\right) h_{i}^{\prime} .
$$

Let $f: \cup \partial Q_{i}^{*} \rightarrow S^{1}$ be the map such that $\left(x^{*}, f\left(x^{*}\right)\right) \in \tau_{1}(K) \subset X_{2}=X_{2}^{*}$ $\times S^{1}$. Then

$$
\operatorname{deg}\left(f \mid l_{i}^{*}\right)=\sum_{j} \varepsilon_{j i}\left(b_{j}+s_{j 1}\right)=\sum_{j} \varepsilon_{j i} \operatorname{deg}\left(f \mid m_{j}^{*}\right)
$$

and by Lemma (5.5) $f$ extends, defining a section $K_{1}$ over $X_{2}^{*}$ which extends $\tau_{1}(K)$.

Now in a similar fashion choose an equivariant homeomorphism $\Phi$ : $\cup Q_{i} \rightarrow \cup Q_{i}$ such that $\Phi^{*}=\mathrm{id}$ and for each $i$,

$$
\Phi\left(q_{i}\right) \sim q_{i} \text { and } \Phi\left(l_{i}\right) \sim l_{i}+\left[\left(r_{i 1}-r_{i 2}\right)+\sum_{j} \varepsilon_{j i}\left(s_{j 2}-s_{j 1}\right)\right] h_{i} .
$$

Then $\Phi(K)$ is a section over $\cup \partial Q_{i}^{*}$ and $\tau_{2} \Phi(K)$ extends to a section $K_{2}$ over all of $X_{2}^{*}$. 
Let $F: X_{2} \rightarrow X_{2}$ be the equivariant homeomorphism such that $F^{*}=$ id and $F\left(K_{1}\right)=K_{2}$. Then $F$ and $\Phi$ combine to give an equivariant homeomorphism $\left(\cup Q_{i}\right) \cup_{\tau_{1}} X_{2} \rightarrow\left(\cup Q_{i}\right) \cup_{\tau_{2}} X_{2}$ which is orientation-preserving since it is the identity on the orbit spaces. We have already seen that up to vertical equivariant isotopy there is just one way to attach $R$ to $X_{2}$. This completes the proof.

6. Equivariant classification. In this section we show that the weighted orbit space $M^{*}$ characterizes $M$ up to orientation-preserving equivariant homeomorphism.

LEMMA (6.1). Let $S^{1}$ act freely on the 3-manifold $A$ with orbit space $A / S^{1} \cong S^{2}$, and let $f: A \rightarrow A$ be an equivariant homeomorphism satisfying $f^{*}=\mathrm{id}$. Then there is a vertical equivariant isotopy of $f$ with the identity of $A$.

Proof. Define $\Phi: A \rightarrow S^{1}$ by $\Phi(z) \cdot z=f(z)$. Then $\Phi$ is continuous and for $\theta \in S^{1}, \Phi(\theta \cdot z) \cdot(\theta \cdot z)=f(\theta \cdot z)=\theta \cdot f(z)=\theta \cdot(\Phi(z) \cdot z)=\Phi(z) \cdot(\theta \cdot z)$. Since the action is free, $\Phi(\theta \cdot z)=\Phi(z)$; so $\Phi$ is constant on orbits. Now $\pi_{1}(A)$ is cyclic, generated by an oriented orbit, so $\Phi_{\#}\left(\pi_{1}(A)\right)=1$. Thus $\Phi$ lifts to a map $\tilde{\Phi}$ to the universal cover:

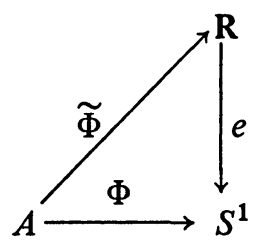

Then $H_{t}(z)=e(t \tilde{\Phi}(z)) \cdot z$ is the desired equivariant isotopy.

THEOREM (6.2). Let $M_{1}$ and $M_{2}$ be oriented simply connected closed 4-manifolds with $S^{1}$-actions. If the weighted orbit spaces of these actions are isomorphic, $M_{1}$ and $M_{2}$ are orientation-preserving equivariantly homeomorphic.

Proof. Identify $M_{1}^{*}$ and $M_{2}^{*}$ by means of the given isomorphism, and let $p_{i}$ : $M_{i} \rightarrow M^{*}$ be the orbit maps. Fix regular neighborhoods $Q_{j}^{*}$ of the weighted circles $L_{j}^{*}$ of $M^{*}$, and choose a regular neighborhood $N^{*}$ of $\left(E^{*} \cup F^{*}\right)-$ $\cup L_{j}^{*}$. We let

$$
X^{*}=\operatorname{cl}\left(M^{*}-\cup Q_{j}^{*}\right), \quad Y^{*}=\operatorname{cl}\left(M^{*}-N^{*}\right),
$$

and

$$
Z^{*}=\operatorname{cl}\left(Y^{*}-\cup Q_{j}^{*}\right)
$$

Since $Y^{*}$ is obtained from $M^{*}$ by removing a collar of $\partial M^{*}$ and a finite number of disjoint 3-disks, $\pi_{1}\left(Y^{*}\right)=1$. 
Consider the principal $S^{1}$-bundles $p_{i} \mid Z_{i} \rightarrow Z^{*}$. It follows from (3.8) that the restriction of $p_{i}$ to each $\partial Q_{j}$ is a trivial $S^{1}$-bundle projection which may be extended over $Q_{j}^{*}$. We obtain in this manner principal $S^{1}$-bundles $p_{i}^{\prime}: Y_{i}^{\prime} \rightarrow$ $Y^{*}$ which restrict over $Z^{*}$ to $p_{i} \mid Z_{i} \rightarrow Z_{i}^{*}$. Let $\xi_{i}$ be the Euler class of $p_{i}^{\prime}$ : $Y_{i}^{\prime} \rightarrow Y^{*}$. We have the exact sequence $H^{2}\left(Y^{*}, \partial Y^{*}\right) \rightarrow H^{2}\left(Y^{*}\right) \rightarrow^{\rho} H^{2}\left(\partial Y^{*}\right)$ and $H^{2}\left(Y^{*}, \partial Y^{*}\right) \approx H_{1}\left(Y^{*}\right)=0$, so $\rho$ is injective. Now

$$
H^{2}\left(\partial Y^{*}\right)=\bigoplus H^{2}\left(B_{k}^{*}\right)
$$

where the $B_{k}^{*}$ are the components of $\partial Y^{*}$, and the $j$ th coordinate of $\rho \xi_{i}$ is $\eta_{i k}$, the Euler class of the restriction of $p_{i}^{\prime}$ to $B_{k}^{*}$. The $\eta_{i k}$ are determined from the weighting of $M_{i}^{*}$, so $\eta_{1 k}=\eta_{2 k}$ for each $k$, and $\rho \xi_{1}=\rho \xi_{2}$. Thus $\xi_{1}=\xi_{2}$ and there is an $S^{1}$-bundle equivalence $Y_{1}^{\prime} \rightarrow Y_{2}^{\prime}$ over $Y^{*}$. This restricts to an equivalence $Z_{1} \rightarrow Z_{2}$ over $Z^{*}$.

The action on $M_{i}$ over a collar of $\partial M^{*}$ is obtained essentially by spreading out the principal orbit bundles and collapsing orbits over $\partial M^{*}$ to points. (See $[1, \mathrm{~V} .10 .1]$.) The action over a regular neighborhood of a weighted point is just the cone of the action over the boundary of that neighborhood. These facts, along with (3.6), (3.9), and (6.1) imply that the equivalence $Z_{1} \rightarrow Z_{2}$ gives rise to an orientation-preserving equivariant homeomorphism $f_{X}: X_{1} \rightarrow$ $X_{2}$.

Also (3.6) provides an orientation-preserving equivariant homeomorphism $f_{Q}: \cup p_{1}^{-1}\left(Q_{j}^{*}\right) \rightarrow \cup p_{2}^{-1}\left(Q_{j}^{*}\right)$ such that $f_{Q}^{*} \mid \partial Q_{j}^{*}=$ id for each $j$. The map $\tau=f_{X}^{-1} f_{Q}: \cup p_{1}^{-1}\left(\partial Q_{j}^{*}\right) \rightarrow \partial X_{1}$ is an equivariant homeomorphism with $\tau^{*}=$ id. Define $h$ by $h\left|\cup Q_{i}=f_{Q}, h\right| X_{1}=f_{X}$. Then $h$ is an orientation-preserving equivariant homeomorphism $\cup Q_{i} \cup_{\tau} X_{1} \rightarrow M_{2}$. But by (5.6) $\cup Q_{i} \cup_{\tau} X_{1}$ is orientation-preserving equivariantly homeomorphic to $M_{1}$.

COROLlaRY (6.3). Orientation-preserving equivariant homeomorphism classes of $S^{1}$-actions on oriented simply connected closed 4-manifolds are in bijective correspondence with isomorphism classes of legally weighted simply connected 3-manifolds.

\section{Torus actions.}

THEOREM (7.1). Let $S^{1}$ act on the simply connected 4-manifold $M$. The action extends to an action of $T^{2}=S^{1} \times S^{1}$ if and only if

(i) $M^{*}$ is not a counterexample to the Poincare conjecture, and

(ii) if $M^{*}$ contains a weighted circle $L^{*}$, then $M^{*} \cong S^{3}, L^{*}=E^{*} \cup F^{*}$, and $L^{*}$ is unknotted in $M^{*}$.

Proof. Suppose that the action extends to a $T^{2}$-action. Applying an automorphism of $T^{2}$, if necessary, we may suppose that the given $S^{1}$-action is the action of the subgroup $0 \times S^{1}$ of $T^{2}$. Then $S^{1} \times 0$ acts effectively on $M /\left(0 \times S^{1}\right)=M^{*}$. This implies (i). If points of $M$ lie in the same $T^{2}$-orbit 
they have the same isotropy groups under the induced $0 \times S^{1}$-action. It follows that $S^{1} \times 0$ pointwise fixes $\left(E^{*} \cup F^{*}\right)-\partial M^{*}$.

Let $p^{\prime}: M^{*} \rightarrow M^{*} /\left(S^{1} \times 0\right)=M / T^{2}$ and $\pi: M \rightarrow M / T^{2}$ be the orbit maps. Then $M / T^{2}$ is a 2-disk whose boundary consists of the $T^{2}$-orbits of $M$ with nontrivial isotropy [10]; so $p^{\prime}\left(E^{*} \cup F^{*}\right)=\pi(E \cup F) \subset \partial\left(M / T^{2}\right)$. This proves (ii).

Conversely, suppose that conditons (i) and (ii) are satisfied. If $M^{*}$ contains no weighted circle let $R^{*}$ be the neighborhood of $E^{*} \cup F^{*}$ which was described in (4.6). By (4.7) there is a 4-manifold $W$ with $T^{2}$-action whose weighted orbit space under the induced $0 \times S^{1}$-action is isomorphic to $R^{*}$. The action of $0 \times S^{1}$ on $\partial W$ is free, and it follows from (5.3) that $\partial W \cong$ $S^{2} \times S^{1}$. Any $T^{2}$-action on $S^{2} \times S^{1}$ has exactly two nonprincipal orbits each of which has a circle group as isotropy group. So both nontrivial isotropy groups on $\partial W$ must be $S^{1} \times 0$.

By condition (i), $X^{*}=\operatorname{cl}\left(M^{*}-R^{*}\right) \cong D^{3}$, so there is an obvious componentwise action of $T^{2}$ on $X^{*} \times S^{1}$. Again the nontrivial isotropy groups on $\partial\left(X^{*} \times S^{1}\right)$ are each $S^{1} \times 0$. If we are careful in the choice of orientation of the $S^{1}$-action on $X^{*}$, there is an orientation-reversing $T^{2}$-equivariant homeomorphism $h: \partial W \rightarrow \partial\left(X^{*} \times S^{1}\right)$. Now $W \cup_{h}\left(X^{*} \times S^{1}\right)$ is easily seen to be a simply connected $T^{2}$-manifold, and the induced $0 \times S^{1}$-action has weighted orbit space isomorphic to $M^{*}$. So $M$ is $S^{1}$-equivariantly homeomorphic to $W \cup_{h}\left(X^{*} \times S^{1}\right)$, and the $S^{1}$-action on $M$ extends to a $T^{2}$-action via this homeomorphism.

If $L^{*}$ is a weighted circle in $M^{*}$ choose a regular neighborhood $Q^{*}$ of $L^{*}$. By (4.4) there is a 4-manifold $W$ with $T^{2}$-action whose weighted orbit space under the induced $0 \times S^{1}$-action is isomorphic to $Q^{*}$, and the $T^{2}$-action restricted to $\partial W \cong T^{3}$ is free. Conditions (i) and (ii) imply that

$$
X^{*}=\operatorname{cl}\left(M^{*}-Q^{*}\right) \cong D^{2} \times S^{1} \text {. }
$$

Letting $S^{1}$ act freely on $X^{*}$ induces a free $T^{2}$-action on $X^{*} \times S^{1}$. Again with careful choice there is an orientation-reversing $T^{2}$-equivariant homeomorphism $g: \partial W \rightarrow \partial\left(X^{*} \times S^{1}\right)$, and we form the $T^{2}$-manifold $W$ $\cup_{g}\left(X^{*} \times S^{1}\right)$. The orbit space $W \cup_{j}\left(X^{*} \times S^{1}\right) / T^{2}$ is a 2-disk and the action is free over the interior of the disk. It follows from [10] that $W \cup_{g}\left(X^{*}\right.$ $\times S^{1}$ ) is a simply connected 4-manifold which as above is $S^{1}$-equivariantly homeomorphic with $M$. This extends the action on $M$.

8. Quadratic forms and homotopy type. Let $S^{1}$ act on the simply connected 4-manifold $M$. We shall compute the quadratic form of $M$ using the information contained in the weighted orbit space $M^{*}$, and this, in turn, will be used to show that $M$ is homotopy equivalent to a connected sum of copies of $S^{4}$, $C P^{2},-C P^{2}$, and $S^{2} \times S^{2}$. 
(8.1) Let $R^{*}$ be the set described in (4.6); so $R^{*}$ is a neighborhood of $\left(E^{*} \cup F^{*}\right)-\cup L_{i}^{*}$ where the $L_{i}^{*}$ are the weighted circles of $M^{*}$. Using (3.6) and (4.7) we identify $R$ with the resultant 4-manifold of an equivariant linear plumbing

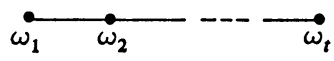

If $\partial M^{*}$ has $m$ components and $\left(F^{*}-\partial M^{*}\right) \cap R^{*}$ contains $l$ points then $t=2 m+l-1$.

The matrix $B_{0}$ of this plumbing is the $t \times t$ matrix with $i j$ th entry

$$
\left(B_{0}\right)_{i j}= \begin{cases}\omega_{i}, & i=j \\ 1, & i=j \pm 1 \\ 0, & \text { otherwise }\end{cases}
$$

since each plumbing involved has sign +1 . Now if $\phi: \mathbf{Z}^{t} \rightarrow \mathbf{Z}^{t}$ is the linear map with matrix $B_{0}$ then $H_{1}(\partial R) \approx \operatorname{coker} \phi[5,8.2]$. But it was shown in (5.3) that $\partial R \cong S^{2} \times S^{1}$; so det $B_{0}=0$.

If $B$ is a square matrix we shall denote by $B^{-}$the matrix obtained from $B$ by striking out the last row and column. If $t \geqslant 2$, then $B_{0}^{-}$is the matrix of the equivariant linear plumbing

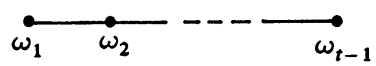

whose resultant manifold we call $V$. According to $[5,8.6], \partial R$ is homeomorphic to the lens space $L(p, q)$ where

$$
\left(\begin{array}{cc}
p & a \\
-q & b
\end{array}\right)=\left(\begin{array}{cc}
-\omega_{t} & 1 \\
-1 & 0
\end{array}\right) \cdots\left(\begin{array}{cc}
-\omega_{1} & 1 \\
-1 & 0
\end{array}\right)=\left(\begin{array}{cc}
-\omega_{t} & 1 \\
-1 & 0
\end{array}\right)\left(\begin{array}{cc}
p^{\prime} & a^{\prime} \\
-q^{\prime} & b^{\prime}
\end{array}\right),
$$

and $\partial V \cong L\left(p^{\prime}, q^{\prime}\right)$. We have $p=0$ since $\partial R \cong S^{2} \times S^{1}$; so $q^{\prime}=-\omega_{\iota} p^{\prime}$. Each matrix in the above product has determinant 1 , hence $p^{\prime}\left(b^{\prime}-\omega_{t} a^{\prime}\right)=$ $p^{\prime} b^{\prime}+a^{\prime} q^{\prime}=1$, and $p^{\prime}= \pm 1$. Thus $\partial V \cong L\left( \pm 1, q^{\prime}\right) \cong S^{3}$. Using $[5,8.2]$ once more we see that $\left|\operatorname{det} B_{0}^{-}\right|$is the order of $H_{1}(\partial V)$, and so $\operatorname{det} B_{0}^{-}= \pm 1$. The zero sections $S_{1}, \ldots, S_{t-1}$ of $Y_{\omega_{1}}, \ldots, Y_{\omega_{t-1}}$ have intersection matrix $B_{0}^{-}$; so considered as elements of $H_{2}(M), S_{1}, \ldots, S_{t-1}$ are independent. If there are no weighted circles in $M^{*}$ then $\chi(F)=2 m+l$; so rk $H_{2}(M)=2 m$ $+l-2=t-1$. In this case $S_{1}, \ldots, S_{t-1}$ also generate $H_{2}(M)$, and $B_{0}^{-}$is the matrix of the quadratic form of $M$.

Now $\partial R^{*}-\partial M^{*} \cong S^{2}$, and attaching a 3-disk to $R^{*}$ along this 2-sphere we obtain a simply connected 3-manifold which we weight by the weights of $R^{*}$. There is a simply connected 4-manifold $Y$ with $S^{1}$-action and $Y^{*}=R^{*}$ $\cup D^{3}$. Since $Y^{*}$ satisfies the hypotheses of (7.1) the $S^{1}$-action on $Y$ extends to 
an action of $T^{2}$. It now follows that $Y$ is a connected sum of copies of $S^{4}$, $C P^{2},-C P^{2}$, and $S^{2} \times S^{2}$ since any simply connected 4-manifold with $T^{2}$-action admits such a connected sum decomposition [10]. The matrix $B_{0}^{-}$ of the quadratic form of $Y$ must then be congruent over $\mathbf{Z}$ to a direct sum of copies of matrices (1), $(-1)$, and $\left(\begin{array}{l}0 \\ 1 \\ 1\end{array}\right)[6]$.

(8.2) Let $L_{1}^{*}, \ldots, L_{k}^{*}$ be the weighted circles of $M^{*}$ with regular neighborhoods $Q_{1}^{*}, \ldots, Q_{k}^{*}$. Fix $j$ and suppose $L_{j}^{*}=\left\{\left(\alpha_{1}, \beta_{1}\right), \ldots,\left(\alpha_{q}, \beta_{q}\right)\right\}$. We may identify $Q_{j}$ with the resultant manifold of an equivariant plumbing according to the graph

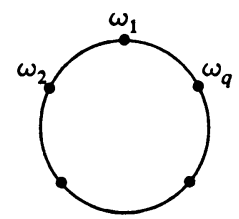

where each plumbing except perhaps for $Y_{\omega_{q}} \square Y_{\omega_{1}}$ has sign +1 . Index the $\omega_{i}$ so that the exceptional orbits lying in the zero section $S_{i}$ of $Y_{\omega_{i}}$ are of type $\mathbf{Z}_{\alpha_{i}}$.

A legally weighted simply connected 3-manifold $X^{*}$, is obtained by embedding $Q_{j}^{*}$ in $S^{3}$ as an unknotted solid torus, and taking $E^{*} \cup F^{*}=L_{j}^{*}$. There is a simply connected 4-manifold $X$ with $S^{1}$-action and weighted orbit space $X^{*}$, and since $X$ has $q$ fixed points, rk $H_{2}(X)=q-2$. Thus $S_{1}, \ldots, S_{q-1}$ are linearly dependent when considered as elements of $H_{2}(X)$; so their intersection matrix $B$ has determinant 0 . But $B$ is the matrix of the linear plumbing

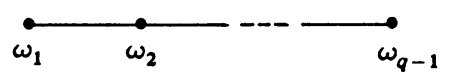

The boundary plumbing yields $S^{2} \times S^{1}[5,8.2]$, and as in (8.1) det $B^{-}= \pm 1$ if $q-1 \geqslant 2$. Again (7.1) applies to $X$, so the action extends to a $T^{2}$-action, and $B^{-}$, the matrix of the quadratic form of $X$, is congruent over $\mathbf{Z}$ to a direct sum of copies of matrices (1), $(-1)$, and $\left(\begin{array}{l}0 \\ 1 \\ 1\end{array}\right)$. Note that if $E^{*} \cup F^{*}=$ $L_{j}^{*}(k=1)$, then $B^{-}$is the matrix of the quadratic form of $M$.

(8.3) Let $L_{j}^{*}$ be as given in (8.2) and suppose that we have $L_{j+1}^{*}=\left\{\left(\alpha_{1}^{\prime}\right.\right.$, $\left.\left.\beta_{1}^{\prime}\right), \ldots,\left(\alpha_{r}^{\prime}, \beta_{r}^{\prime}\right)\right\}$. As before we view $Q_{j+1}$ as the resultant manifold of an equivariant plumbing with zero sections $S_{1}^{\prime}, \ldots, S_{r}^{\prime}$. In $M$ we let $x \in S_{q-1} \cap$ $S_{q} \subset L_{j}$ and $y \in S_{r}^{\prime} \cap S_{1}^{\prime} \subset L_{j+1}$. Choose an arc $\Gamma_{j}^{*}$ in $M^{*}$ which has endpoints $x^{*}$ and $y^{*}$ and such that $\Gamma_{j}^{*}-\left\{x^{*}, y^{*}\right\} \subset P^{*}$. Then $\Gamma_{j}$ is a 2-sphere in $M$. There is a 4-disk $D$ which is a linear slice to the action at $x$, and we may suppose $\partial D \cap \Gamma_{j}$ is a single orbit. The intersection number $\Gamma_{j} \cdot S_{q-1}$ is the linking number in $\partial D$ of the principal orbit $\Gamma_{j} \cap \partial D$ with the $\mathbf{Z}_{\alpha_{q-1}}$-orbit $S_{q-1} \cap \partial D$. Thus $\Gamma_{j} \cdot S_{q-1}=\delta \alpha_{q}$ for $\delta= \pm 1$. Also $\Gamma_{j} \cdot S_{q}=\varepsilon \alpha_{q-1}$, 
$\varepsilon= \pm 1$. Similar considerations show that $\gamma_{j}=\Gamma_{j} \cdot \Gamma_{j}= \pm \alpha_{q-1} \alpha_{q} \pm \alpha_{r}^{\prime} \alpha_{1}^{\prime}$. This determines $\gamma_{j}$ modulo 2 which is all that will be needed. We let $\lambda_{j}$ be $\Gamma_{j} \cdot S_{1}^{\prime}$.

Since $\alpha_{q-1}$ and $\alpha_{q}$ are relatively prime we may choose integers $m$ and $n$ with $\varepsilon m \alpha_{q-1}+\delta n \alpha_{q}=1$. If we let $T_{j}$ be the 2-cycle $n S_{q-1}+m S_{q}$ on $Q_{j}$ then $\Gamma_{j} \cdot T_{j}=1$. The intersection matrix $B_{j}$ of $S_{1}, \ldots, S_{q-2}, T_{j}$ has determinant 0 since these elements are dependent in $H_{2}(X)$, and if $q>3$ the $(s-t)$ th entry of $B_{j}$ is

$$
\left(B_{j}\right)_{s t}=\left\{\begin{array}{l}
\omega_{s}, \quad s=t \leqslant q-2, \\
m^{2} \omega_{q}+n^{2} \omega_{q-1}+2 n m, \quad s=t=q-1, \\
1, \quad(s, t)=(s, s \pm 1) \text { and } s \neq q-1, t \neq q-1, \\
\pm m, \quad(s, t)=(1, q-1) \text { or }(q-1,1) \\
n, \quad(s, t)=(q-2, q-1) \text { or }(q-1, q-2), \\
0, \quad \text { otherwise. }
\end{array}\right.
$$

If $q=2$ then $B_{j}=(0)$, and if $q=3$ then

$$
B_{j}=\left(\begin{array}{cc}
\omega_{1} & n \pm m \\
n \pm m & m^{2} \omega_{3}+n^{2} \omega_{2}+2 n m
\end{array}\right) .
$$

Now $B_{j}{ }^{-}$is the matrix $B^{-}$of (8.2); so $B_{j}^{-}$is congruent over $\mathbf{Z}$ to a direct sum of matrices $(1),(-1)$, and $\left(\begin{array}{l}0 \\ 1 \\ 1\end{array}\right)$.

(8.4) Suppose that $R^{*} \neq \varnothing$ and that $S_{1}, \ldots, S_{t}$ are the zero sections as in (8.1). Further suppose that we have $L_{1}^{*}=\left\{\left(\alpha_{1}^{\prime}, \beta_{1}^{\prime}\right), \ldots,\left(\alpha_{r}^{\prime}, \beta_{r}^{\prime}\right)\right\}$ with zero sections $S_{i}^{\prime}$. Let $x \in\left(S_{t}-S_{t-1}\right) \cap F$ and $y \in S_{r}^{\prime} \cap S_{1}^{\prime}$. If $\Gamma_{0}^{*}$ is an arc in $M^{*}$ with endpoints $x^{*}$ and $y^{*}$ such that $\Gamma_{0}^{*}-\left\{x^{*}, y^{*}\right\} \subset P^{*}$ then $\Gamma_{0}$ is a 2-sphere in $M$. We have $\Gamma_{0} \cdot S_{t}=1$ and $\gamma_{0}=\Gamma_{0} \cdot \Gamma_{0}= \pm \alpha \pm \alpha_{r}^{\prime} \alpha_{1}^{\prime}$ where $S_{t}$ has orbits of type $\mathbf{Z}_{\alpha}$. (We let $\alpha=0$ if $S_{t} \subset F$ and $\alpha=1$ if $S_{t} \cap P \neq \varnothing$.) Finally, let $\lambda_{0}=\Gamma_{0} \cdot S_{1}^{\prime}$.

(8.5) We now have $B_{0}$, an intersection matrix of 2-cycles in $R$, and $B_{1}, \ldots, B_{k}$, intersection matrices of 2-cycles in $Q_{1}, \ldots, Q_{k}$. For each $i=$ $0, \ldots, k$ let $d_{i}$ denote the dimension of the matrix $B_{i}$. Define the matrix $C_{i}^{\prime}=B_{i} \oplus\left(\gamma_{i}\right) \oplus B_{i+1} \oplus \cdots \oplus B_{k-1} \oplus\left(\gamma_{k-1}\right) \oplus B_{k}^{-}$, for $i=0, \ldots, k-1$ $\left(i=1, \ldots, k-1\right.$ if $\left.R^{*}=\varnothing\right)$ where $\left(\gamma_{i}\right)$ is the $1 \times 1$ matrix with entry $\gamma_{i}$. Further for $i=0, \ldots, k-1$ define $C_{i}^{\prime \prime}=O_{i} \oplus H_{i} \oplus O_{i+1}^{-} \oplus \cdots \oplus O_{k-1}^{-}$ $\oplus H_{k-1} \oplus O_{k}^{-}$where $H_{j}$ is the $3 \times 3$ matrix

$$
\left[\begin{array}{ccc}
0 & 1 & 0 \\
1 & 0 & \lambda_{j} \\
0 & \lambda_{j} & 0
\end{array}\right]
$$


and $O_{j}$ is the $\left(d_{j}-1\right) \times\left(d_{j}-1\right)$ zero matrix. Also let $C_{k}^{\prime}=B_{k}^{-}$and $C_{k}^{\prime \prime}=$ $O_{k}$. The square matrices $C_{i}^{\prime}$ and $C_{i}^{\prime \prime}$ have the same dimension so we may form $C_{i}=C_{i}^{\prime}+C_{i}^{\prime \prime}$.

Define $C=C_{0}$ if $R^{*} \neq \varnothing$ and $C=C_{1}$ if $R^{*}=\varnothing$. Then $C$ is the intersection matrix of 2-cycles examined in (8.1)-(8.4). It is easy to check that $C$ has rk $H_{2}(M)$ rows and columns.

THEOREM (8.6). The quadratic form of $M$ has matrix $C$.

THEOREM (8.7). $M$ is homotopy equivalent to a connected sum of copies of $S^{4}$, $C P^{2},-C P^{2}$, and $S^{2} \times S^{2}$.

Proof. We prove both theorems by showing that $C$ is congruent over $\mathbf{Z}$ to a direct sum of matrices $(1),(-1)$, and $\left(\begin{array}{ll}0 & 1 \\ 1 & 0\end{array}\right)$. This implies that $\operatorname{det} C= \pm 1$, so $H_{2}(M)$ is generated by the 2-cycles for which $C$ is the intersection matrix. This proves (8.6) and then (8.7) follows from the homotopy type classification of simply connected 4-manifolds [6].

Our proof proceeds by induction on $n(C)$, where $n(C)=k+1$ if $R^{*} \neq \varnothing$ and $n(C)=k$ if $R^{*}=\varnothing$. If $n(C)=1$ then $(8.1)-(8.3)$ prove that $C$ is congruent over $\mathbf{Z}$ to a direct sum of matrices $(1),(-1)$, and $\left(\begin{array}{ll}0 & 1 \\ 1 & 0\end{array}\right)$.

So suppose $n(C)>1$, and let $\varepsilon=0$ if $R^{*} \neq \varnothing$ and $\varepsilon=1$ if $R^{*}=\varnothing$. The operation of adding an integral constant times row $i$ to row $j$ and then that constant times column $i$ to column $j$ preserves the congruence class over $\mathbf{Z}$ of an integral matrix. Let us call this an elementary operation. In (8.1) and (8.2) we saw that det $B_{\varepsilon}=0$. So if $d_{\varepsilon}$, the dimension of $B_{\varepsilon}$, is 1 then $B_{\varepsilon}=(0)$. In this case $C$ has the form

$$
\left(\begin{array}{ccc}
0 & 1 & 0 \\
1 & \gamma_{\epsilon} & \lambda_{\epsilon} \\
0 & \lambda_{\epsilon} & \\
0 & \nVdash
\end{array}\right)
$$

where the lower right-hand corner matrix is $C_{\varepsilon+1}$. The elementary row operation $-\lambda_{\varepsilon}$ times the first added to the third yields the matrix

$$
\left(\begin{array}{ll}
0 & 1 \\
1 & \gamma_{\varepsilon}
\end{array}\right) \oplus C_{\varepsilon+1}
$$

which is thus congruent to $C$.

If $d_{\varepsilon} \geqslant 2$ and $d=d_{\varepsilon}-1$ there is a $d \times d$ integral invertible matrix $E$ such that $E^{\prime} B_{\varepsilon}-E$ is a direct sum of matrices (1), $(-1)$, and $\left(\begin{array}{l}0 \\ 1 \\ 1\end{array}\right)$. Let $F$ be the matrix $E \oplus I$ where $I$ is the identity matrix so that $E \oplus I$ has the same dimension as $C$. Then 


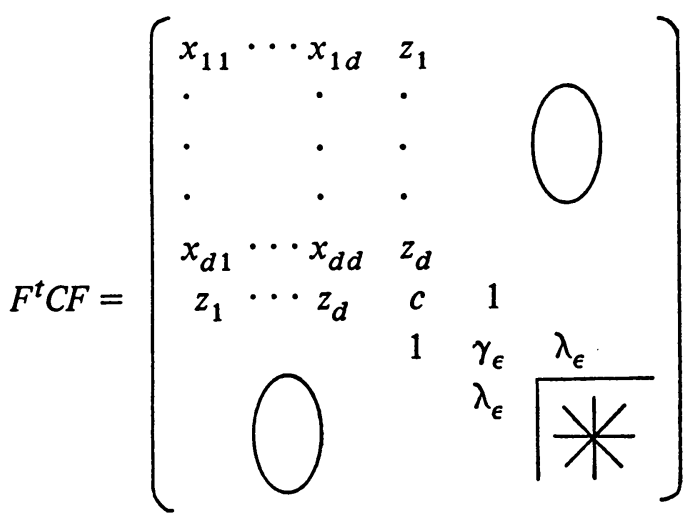

where the lower right-hand corner matrix is $C_{\varepsilon+1}$ and $x_{i j}$ is the $i j$ th entry of $E^{t} B_{\mathrm{e}}^{-} E$.

The rows (and columns) of $E^{t} B_{\varepsilon}{ }^{-} E$ are linearly independent, and each consists of $d-1$ zeros and one 1 . Thus a sequence of elementary operations may be performed on $F^{t} C G$ to yield the congruent matrix

$$
A_{1}=\left[\begin{array}{ccccc}
x_{11} & \cdots & x_{i d} & & \\
\cdot & \cdot & & \\
\cdot & \cdot & & \\
x_{d 1} \cdots x_{d d} & & & \\
& & c^{\prime} & 1 & \\
& 1 & \gamma_{\epsilon} & \lambda_{\epsilon} \\
& & \lambda_{\epsilon} & \searrow
\end{array}\right]
$$

where the lower right-hand corner is still $C_{\varepsilon+1}$.

If $G=E \oplus$ (1) then

$$
G^{t} B_{\varepsilon} G=\left(\begin{array}{llll}
x_{11} & \cdots & x_{1 d} & z_{1} \\
\cdot & & \cdot & \cdot \\
\cdot & & \cdot & \cdot \\
x_{d 1} & \cdots & x_{d d} & z_{d} \\
z_{1} & \cdots & z_{d} & c
\end{array}\right) .
$$

Performing the same elementary operations on $G^{t} B_{\varepsilon} G$ that were performed on $F^{t} C F$ results in the matrix $E^{t} B_{\varepsilon}-E \oplus\left(c^{\prime}\right)$, which is congruent over $\mathbf{Z}$ to $B_{\varepsilon}$. Thus $0=\operatorname{det} B_{\varepsilon}=c^{\prime} \operatorname{det}\left(E^{t} B_{\varepsilon}^{-} E\right)= \pm c^{\prime}$. 
Now the elementary operation $-\lambda_{\varepsilon}$ times the $d_{\varepsilon}$ th added to the $\left(d_{\varepsilon}+2\right)$ nd performed on $A_{1}$ yields the congruent matrix

$$
A_{2}=E^{t} B_{\varepsilon}^{-} E \oplus\left(\begin{array}{cc}
0 & 1 \\
1 & \gamma_{\varepsilon}
\end{array}\right) \oplus C_{\varepsilon+1}
$$

Note that $\left(\begin{array}{ll}0 & 1 \\ 1 & \gamma_{\varepsilon}\end{array}\right)$ is congruent to $\left(\begin{array}{ll}0 & 1 \\ 1 & 0\end{array}\right)$ if $\gamma_{\varepsilon}$ is even and to (1) $\oplus(-1)$ if $\gamma_{\varepsilon}$ is odd. There is clearly a simply connected 4-manifold with $S^{1}$-action whose matrix associated by $(8.5)$ is $C_{\varepsilon+1}$. Since $n\left(C_{\varepsilon+1}\right)<n(C)$, the induction is completed.

(8.8) There are easily constructed examples of $S^{1}$-actions on simply connected 4-manifolds which are not equivariant connected sums of homotopy $C P^{2}$ 's, $-C P^{2}$ 's, and $S^{2} \times S^{2}$ 's. The weighted orbit space $S^{3}$ with three weighted circles each linking the other two results in such an example.

\section{REFERENCES}

1. G. E. Bredon, Introduction to compact transformation groups, Academic Press, New York, 1972.

2. W. Browder, Surgery on simply-connected manifolds, Springer-Verlag, Berlin and New York, 1972. MR 50 \#11272.

3. P. T. Church and K. Lamotke, Almost free actions on manifolds, Bull. Austral. Math. Soc. 10 (1974), 177-196. MR 51 \#6859. 63-70.

4. R. Fintushel, Locally smooth circle actions on homotopy 4-spheres, Duke Math. J. 43 (1976),

5. F. Hirzebruch, W. D. Neumann and S. S. Koh, Differentiable manifolds and quadratic forms, Dekker, New York, 1971. MR 49 \#6250.

6. J. Milnor, On simply connected 4-manifolds, Sympos. internac. de topologia algebraica, Universidad Nac. Autónoma de México and UNESCO, Mexico City, 1958, pp. 122-128. MR 21 \#2240.

7. D. Montgomery and C. T. Yang, Groups on $S^{n}$ with principal orbits of dimension $n-3$, Illinois J. Math. 4 (1960), 507-517. MR 23 \#A3199.

8. P. Orlik, Seifert manifolds, Lecture Notes in Math., vol. 291, Springer-Verlag, Berlin and New York, 1972.

9. P. Orlik and F. Raymond, Actions of SO(2) on 3-manifolds, Proc. Conf. on Transformation Groups (New Orleans, La., 1967), Springer-Verlag, Berlin and New York, 1968, pp. 297-318. MR 41 \#7717.

10. __ Actions of the torus on 4-manifolds. I, Trans. Amer. Math. Soc. 152 (1970), 531-559. MR 42 \#3808.

11. H. Seifert, Topologie dreidimensionaler gefaserter Räume, Acta Math. 60 (1933), 147-238.

12. E. Spanier, Algebraic topology, McGraw-Hill, New York, 1966. MR 35 \#1007.

Department of Mathematics, Tulane University, New Orleans, Louisiana 70118 